\title{
Combustion model reduction for diesel engine control design
}

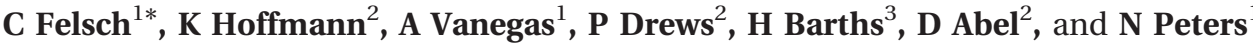 \\ ${ }^{1}$ Institut für Technische Verbrennung, RWTH Aachen University, Aachen, Germany \\ ${ }^{2}$ Institut für Regelungstechnik, RWTH Aachen University, Aachen, Germany \\ ${ }^{3}$ DRE Turbochargers, GM Powertrain, USA
}

The manuscript was accepted after revision for publication on 4 August 2009.

DOI: 10.1243/14680874JER04509

\begin{abstract}
The subject of this work is the derivation of a simulation model for premixed charge compression ignition (PCCI) combustion that can be used in closed-loop control development. For the high-pressure part of the engine cycle, a detailed three-dimensional computational fluid dynamics model is reduced to a stand-alone multi-zone chemistry model. This multi-zone chemistry model is extended by a mean value model accounting for the gas exchange losses. The resulting model is capable of describing PCCI combustion with stationary exactness, and is at the same time very economic with respect to computational costs. The model is further extended by the identified system dynamics that influence the stationary inputs. For this purporse, a Wiener model is set up that uses the stationary model as a non-linear system representation. In this way, a dynamic non-linear model for the representation of the controlled plant diesel engine is created.
\end{abstract}

Keywords: PCCI combustion, computational fluid dynamics, combustion model reduction, stand-alone multi-zone model, closed-loop control

\section{INTRODUCTION}

There is a strong demand to explore new combustion concepts capable of meeting stringent emission standards, such as Tier 2 Bin 5 on the North American market and EURO VI on the European market. Premixed charge compression ignition (PCCI), or premixed compression ignition, as it is sometimes referred to, has emerged as an interesting alternative to conventional diesel combustion in the part-load operating range [1-3]. This concept involves relatively early injection timings, high external exhaust gas recirculation (EGR) rates, and cooled intake air, leading to a low-temperature combustion (LTC) process with low $\mathrm{NO}_{x}$ and particulate emissions. A general review of LTC processes can be found in reference [4]. However, in contrast to

*Corresponding author: Institut für Technische Verbrennung, RWTH Aachen University, Templergraben 64, D-52056 Aachen, Germany.

email: c.felsch@itv.rwth-aachen.de conventional diesel combustion, with early directinjection LTC it can be difficult to prevent combustion from occurring before top dead centre (TDC), which often increases noise and reduces engine efficiency. Sophisticated closed-loop control of the combustion process is one means to overcome this difficulty. Against this background, this paper proposes a first step towards the development of a controller for the PCCI combustion process.

The most important characteristics for the operation of an internal combustion engine are the engine's load and its combustion efficiency. The former is directly dependent on the indicated mean effective pressure (IMEP), the latter can be characterized by the centre of combustion, where 50 per cent of the total injected fuel mass is burned (CA50). The engine's load is set by the driver in an automobile application. For CA50, a set point can be determined which is dependent on the operating point and gives the best combustion efficiency. These two variables are the main focus of the controller to be developed. For influencing the 
process, the start of injection (SOI), the external EGR rate, and the total fuel mass injected are suitable actors.

In the recent past, several efforts have been reported in the literature that aim at controlling engine combustion. Among these, references [5-11] can be mentioned. The standard procedure for creating a controller includes the modelling part as the first step [12-16]. Often these models differ in several aspects from models widely used for gathering a deeper understanding of combustion details, like three-dimensional (3D) computational fluid dynamics (CFD) models [17-20]. From the viewpoint of automatic control, the dynamics describing the dependency of the system's outputs (IMEP, CA50) on the actors (SOI, external EGR rate, and total fuel mass injected) is of highest priority. Nevertheless, stationary exactness of the model is also important. Another requirement is an acceptable calculation speed, as this model is usually applied in dynamic closed-loop simulations.

This paper presents a new approach to the development of a simulation model for use in closed-loop control development. A detailed 3D CFD model is reduced to a computationally efficient stand-alone multi-zone model. The stand-alone multi-zone model covers the non-linear dependencies within the high-pressure part of the engine cycle with stationary exactness. This model is extended by a physically inspired description of the gas exchange part of the engine cycle. For use in closed-loop simulations, the system's dynamics have to be covered. For this reason, the stationary model is further extended by identifying system dynamics that influence the stationary inputs. In this manner, a stationary exact model is extended to a Wienertype model with a static part describing the nonlinearities and an upstream part describing the system's dynamics. This novel procedure integrates the detailed knowledge from combustion simulation tools into closed-loop control and establishes a broad field of possibilities for testing completely new controlled process variables.

The paper is arranged as follows: the first section deals with the combustion modelling approach employed in the present investigation. The reduction of the 3D CFD model to a computationally efficient stand-alone multi-zone chemistry model is described. After this, the four-cylinder test engine, the investigated operating points, as well as the corresponding numerical setups, are presented. The next section contains the stationary validation of the employed combustion modelling approach. Subse- quently, the physically inspired description of the gas exchange part of the engine cycle is put forward. Afterwards, the system's dynamics are identified. Following this, the integrated model composed of all three model parts (stand-alone multi-zone model, gas exchange model, and dynamic time response) is validated against transient experimental data. Finally, the conclusions and major findings from the study are summarized and an outlook to future work is given.

\section{COMBUSTION MODEL FORMULATION}

\subsection{Multi-zone chemistry model}

\subsubsection{Multi-zone modelling overview}

In homogeneous charge compression ignition (HCCI) or PCCI engines, where substantial premixing occurs, the cylinder charge may be divided into several distinct regions, each characterized by a certain thermodynamic state. A major requirement is for the variance within each individual zone to be small, since the multi-zone chemistry code solves only for the average values provided.

Several multi-zone approaches have been proposed in the literature. Aceves et al. [21] and Aceves et al. [22] used zones based on the temperature to account for thermal gradients inside the cylinder of heavy-duty truck engines running in HCCI mode on natural gas and propane. Babajimopoulos et al. [23] used equivalence ratio, temperature, and EGR to define the zones in investigating various variable valve actuation strategies in a heavy-duty truck engine fuelled by natural gas. In all these studies, the zones were initialized based on 3D CFD calculations of the flow up to a certain crank angle at which chemical reactions start to occur. The mass of each zone was primarily governed by the given temperature distribution in the cylinder, and no mixing occurred between the zones in the multi-zone chemistry code. In the study by Babajimopoulos et al. [23], each temperature zone was divided into three equivalence ratio zones of equal mass.

In a more recent publication, Babajimopoulos et al. [24] extended the sequential approach presented in reference [23] to a fully coupled CFD and multizone model with detailed chemical kinetics. The multi-zone model communicated with the CFD code at each computational time step, and the composition of the CFD cells was mapped back and forth between the CFD code and the multi-zone model. 
This approach was validated against experimental data in references [25] and [26].

\subsubsection{Multi-zone model}

The multi-zone model employed in the current study is X0D, a zero-dimensional chemistry solver based on multiple zero-dimensional reactors. XOD was developed internally at General Motors R\&D by Hardo Barths, Tom Sloane, and Christian Hasse, and was first described in Hergart et al. [27] and Felsch et al. [28].

The equations governing species mass fractions, temperature, and pressure change in the multi-zone chemistry code are

$$
\begin{aligned}
& \frac{\mathrm{d} Y_{i j}}{\mathrm{~d} t}-\frac{1}{m_{i}} \sum_{k=1}^{n z} \dot{m}_{i k}\left(Y_{k j}^{\mathrm{ex}}-Y_{i j}\right)-\frac{\dot{\omega}_{i j}}{\rho_{i}}-\frac{\dot{\rho}_{i j}^{\mathrm{s}}}{\rho_{i}}=0 \\
& \frac{\mathrm{d} T_{i}}{\mathrm{~d} t}-\frac{1}{m_{i} \times \bar{c}_{\mathrm{p} i}} \sum_{k=1}^{n z} \dot{m}_{i k}\left(\bar{h}_{k}^{\mathrm{ex}}-\bar{h}_{i}\right) \\
& \quad+\frac{1}{m_{i} \times \bar{c}_{\mathrm{p} i}} \sum_{j=1}^{n s p} h_{i j} \sum_{k=1}^{n z} \dot{m}_{i k}\left(Y_{k j}^{\mathrm{ex}}-Y_{i j}\right) \\
& \quad+\frac{1}{\rho_{i} \times \bar{c}_{\mathrm{p} i}} \sum_{j=1}^{n s p} h_{i j} \times \dot{\omega}_{i j}-\frac{1}{\rho_{i} \times \bar{c}_{\mathrm{p} i}} \frac{\mathrm{d} p}{\mathrm{~d} t} \\
& \quad-\frac{1}{\rho_{i} \times \bar{c}_{\mathrm{p} i}} \sum_{j=1}^{n s p} \dot{\rho}_{i j}^{\mathrm{s}} \times \Delta h_{\mathrm{v} j}+\frac{1}{m_{i} \times \bar{c}_{\mathrm{p} i}} \dot{Q}_{\mathrm{wall}, i}=0
\end{aligned}
$$

and

$$
\begin{aligned}
\frac{\mathrm{d} p}{\mathrm{~d} t} & =-\frac{p}{V} \frac{\mathrm{d} V}{\mathrm{~d} t} \\
& +\frac{p}{V} \sum_{i=1}^{n z} V_{i}\left(\frac{1}{m_{i}} \frac{\mathrm{d} m_{i}}{\mathrm{~d} t}+\frac{1}{T_{i}} \frac{\mathrm{d} T_{i}}{\mathrm{~d} t}+\bar{W}_{i} \sum_{j=1}^{n s p} \frac{1}{W_{j}} \frac{\mathrm{d} Y_{i j}}{\mathrm{~d} t}\right)
\end{aligned}
$$

In equation (1), $Y_{i j}$ denotes the mass fraction of species $j$ in zone $i$, and $\dot{\omega}_{i j}$ is the corresponding chemical source term. $\dot{\rho}_{i j}^{\mathrm{s}}$ accounts for the source term due to fuel vaporization. It is zero for all species except the fuel itself $\left(\dot{\rho}_{i j}^{\mathrm{s}}=0, j \neq\right.$ fuel $)$. The second term on the left-hand side of equation (1) describes the mass exchange between the zones, where $\dot{m}_{i k}$ is the rate at which mass is transported between zones $i$ and $k$. Thereby, $n z$ stands for the total number of zones. Similarly, in equation (2) the second and third terms on the left-hand side represent the enthalpy exchange between zones due to enthalpy stratification between zones and due to species stratification between zones, respectively. $\bar{c}_{\mathrm{p} i}$ is the heat capacity at constant pressure of zone $i, \Delta h_{\mathrm{vj}}$ denotes the latent heat of vaporization of species $j$, and $\dot{Q}_{\text {wall, } i}$ is the wall heat transfer of zone $i$. In equations (2) and (3), nsp accounts for the number of species employed in the underlying chemical mechanism. The equation of state is used to derive equation (3), which solves for the pressure across the zones. Through this equation, all zones are thermodynamically coupled with each other. Wall heat transfer is described as

$$
\dot{Q}_{\mathrm{wall}, i}=\sum_{l=1}^{n_{\mathrm{wall}}} A_{\mathrm{wall}, l, i} \times h_{\mathrm{wall}, l, i}\left(T_{i}-T_{\mathrm{wall}, l}\right)
$$

where $n_{\text {wall }}$ denotes the total number of walls in the engine, $A_{\text {wall }, l, i}$ the area of wall $l$ belonging to zone $i$, $h_{\text {wall }, l, i}$ the heat transfer coefficient of zone $i$ to wall $l$, $T_{i}$ the temperature of zone $i$, and $T_{\text {wall, } l}$ the temperature of wall $l$.

The mixing in the multi-zone model is accounted for by allowing the different zones to exchange mass and energy with each other, in addition to the interaction through the pressure. They exchange their scalar quantities (species composition and enthalpy) based on the rate at which they exchange their mass. The mass of each zone is kept constant and the model can accommodate any number of zones.

The multi-zone model is written in FORTRAN 77. Equations (1), (2), and (3) are numerically solved using the differential/algebraic system solver DASSL [29].

\subsubsection{Chemistry model}

A major aspect in modelling diesel engine combustion is the treatment of the chemistry. Combustion chemistry in X0D is described by a detailed chemical mechanism that comprises 59 elementary reactions among 38 chemical species. This mechanism describes low-temperature auto-ignition and combustion of n-heptane, which serves as a surrogate fuel for diesel in this work. In addition, it accounts for thermal NO formation. The chemical mechanism for n-heptane was constructed by Peters et al. [30]. The NO-submechanism, which is part of the full mechanism, is the extended Zeldovich mechanism [31].

\subsection{CFD code}

The CFD code used in this work is AC-FluX (formerly known as GMTEC), a flow solver based on finite volume methods [32] that employs unstructured, 
mostly hexahedral meshes. AC-FluX solves for the partial differential equations for continuity, the Navier-Stokes equations, an equation for the total enthalpy, and two equations to account for the turbulence (k-epsilon-model). The spray liquid phase is modelled using the discrete droplet model, which approximates the spray by a Monte-Carlo simulation. The gas phase and the liquid phase are coupled through source terms in the governing equations for the gas phase. AC-FluX is documented by Khalighi et al. [33] and by Ewald et al. [34]. A detailed description of the spray modelling in ACFluX can be found in reference [35].

The composition of the charge is represented by four so-called active species streams, which are the mass fraction of fuel $\tilde{Y}_{\text {fuel }}$, the mass fraction of air $\tilde{Y}_{\text {air }}$, the mass fraction of combustion products $\left(\mathrm{CO}_{2}\right.$ and $\left.\mathrm{H}_{2} \mathrm{O}\right) \tilde{Y}_{\text {products}}$, and the mass fraction of residuals $\tilde{Y}_{\mathrm{EGR}}$. The corresponding transport equation is

$$
\frac{\partial \bar{\rho} \tilde{Y}_{j}}{\partial t}+\frac{\partial \bar{\rho} \tilde{v}_{i} \tilde{Y}_{j}}{\partial x_{i}}=\frac{\partial}{\partial x_{i}}\left(\bar{\rho} D_{j} \frac{\partial \tilde{Y}_{j}}{\partial x_{i}}\right)+\bar{\rho} \dot{\omega}_{j}+\dot{\rho}_{j}^{\mathrm{s}}
$$

In equation (5), $\tilde{Y}_{j}$ denotes active species stream $j, \bar{\rho}$ the density, $\tilde{v}$ the velocity, $D_{j}$ the diffusion coefficient of active stream $j$, and $\dot{\omega}_{j}$ the chemical source term of active stream $j$. The source term for the residuals is identical zero $\left(\dot{\omega}_{\mathrm{EGR}}=0\right)$, because they are assumed to be chemically inert. The residuals are therefore an indicator for dilution in the CFD code. The term $\dot{\rho}_{j}^{\mathrm{s}}$ represents the source term due to fuel vaporization. It is zero for all species except the fuel itself $\left(\dot{\rho}_{j}^{\mathrm{s}}=0, j \neq\right.$ fuel $)$.

For the interactive coupling to the multi-zone model XOD, two additional scalars are introduced. These are the fuel mixture fraction $Z_{\text {fuel }}$ and an artificial scalar $S$. The fuel mixture fraction $Z_{\text {fuel }}$ is the dominant quantity for the description of nonpremixed combustion and can be related to the commonly used equivalence ratio $\phi$ or the combustion-air ratio $\lambda$, respectively, according to

$$
\phi=\frac{1}{\lambda}=\frac{Z_{\text {fuel }}}{1-Z_{\text {fuel }}} \frac{\left(1-Z_{\text {fuel }, \text { st }}\right)}{Z_{\text {fuel, st }}}
$$

where $Z_{\text {fuel, st }}$ is the fuel mixture fraction at stoichiometric mixture [36]. In the CFD code, a transport equation for the mean fuel mixture fraction $\tilde{Z}_{\text {fuel }}$ is solved, namely

$$
\frac{\partial \bar{\rho} \tilde{Z}_{\text {fuel }}}{\partial t}+\frac{\partial \bar{\rho} \tilde{v}_{i} \tilde{Z}_{\text {fuel }}}{\partial x_{i}}=\frac{\partial}{\partial x_{i}}\left(\bar{\rho} D_{Z} \frac{\partial \tilde{Z}_{\text {fuel }}}{\partial x_{i}}\right)+\dot{\rho}_{\text {fuel }}^{\mathrm{s}}
$$

Similar to equation (5), the term $\dot{\rho}_{\text {fuel }}^{\mathrm{s}}$ represents the source term due to fuel evaporation.

In addition to the transport equation for the mean fuel mixture fraction $\tilde{Z}_{\text {fuel }}$, a generic convectiondiffusion equation without source terms is considered for the mean artificial scalar $\tilde{S}$. This transport equation can be written as

$$
\frac{\partial \bar{\rho} \tilde{S}}{\partial t}+\frac{\partial \bar{\rho} \tilde{v}_{i} \tilde{S}}{\partial x_{i}}=\frac{\partial}{\partial x_{i}}\left(\bar{\rho} D_{S} \frac{\partial \tilde{S}}{\partial x_{i}}\right)
$$

It is assumed that the diffusion coefficients $D_{j}, D_{Z}$, and $D_{S}$ are identical.

Solving equations (7) and (8) is mandatory in order to establish the interactive coupling of the CFD code AC-FluX and the multi-zone model X0D. The interactive coupling is described in the following subsection.

\subsection{Interactive coupling of the CFD code and multi-zone model}

The interactive coupling (two-way-coupling) of CFD codes and zero-dimensional multi-zone codes was introduced by Barths et al. [37]. It is also described in Felsch et al. [38]. In this subsection, the main aspects are discussed. The basic concept of the interactive coupling is explained in section 2.3.1.

After its initialization, the interactive coupling consists of one information stream which is fed from the CFD code to the multi-zone model. Mixing effects are introduced into the multi-zone model based on the CFD solution of the phase variable. Vice versa, one information stream is fed from the multizone model to the CFD code in order to keep the heat release consistent between both codes. These issues are addressed in sections 2.3.2, 2.3.3, and 2.3.4. Section 2.3.5 deals with the treatment of source terms within the interactive coupling.

\subsubsection{Basic concept}

The interactive coupling of a CFD code and a multizone code based on multiple zero-dimensional reactors allows for an accurate modelling of PCCI combustion. It combines 3D flow field information with detailed chemistry. The basic concept is similar to that of a probability density function (PDF) method or a representative interactive flamelet (RIF), where the chemistry is solved in phase space instead of the physical space in the CFD code. In the PDF method, for example, each particle in phase 
space can be considered as a single zone. The physical domain in the CFD code is subdivided into multiple zones based on the fuel mixture fraction. Each zone in the CFD code is represented by a corresponding one in the zero-dimensional code. The zero-dimensional code solves the chemistry for each zone, and the heat release is fed back into the CFD code. The difficulty in facing this kind of methodology is to keep the thermodynamic state of each zone consistent between the CFD code and the zero-dimensional code after the initialization of the zones in the multi-zone code has taken place. The thermodynamic state of each zone changes in time due to mixing and source terms (e.g. chemical heat release, vaporization of fuel, and wall heat transfer). An elaborate mixing model and appropriate treatments of the chemistry and the source terms keep the thermodynamic state of the zones in the CFD code and the zero-dimensional code identical.

\subsubsection{Initialization}

In order to construct the mass distribution function and subdivide it into discrete zones in phase space, the computational cells in the physical CFD domain are sorted in ascending order in terms of the mean fuel mixture fraction $\tilde{Z}_{\text {fuel }}$. The computational cells are then assigned to the zones so that these have equal mass. This is not a requirement, but it simplifies the derivation and implementation. This procedure is repeated at each time step of the CFD code. Only once, however, at a certain crank angle prior to any chemical reactions starting to occur, a corresponding set of zones defined by specific fuel masses, temperatures, and residual mass fractions is initialized in the multi-zone model X0D.

At the initialization point, the distribution of the mean fuel mixture fraction $\tilde{Z}_{\text {fuel }}$ is mapped on the mean artificial scalar $\tilde{S}$. From this transition point on, the sorting procedure is continued based on the mean artificial scalar $\tilde{S}$. This is done in order to account for fuel injection and vaporization being moderately coupled with combustion chemistry during PCCI combustion (in contrast to being completely uncoupled during HCCI combustion). Fuel vaporization also takes place after initialization of the interactive coupling. In this case, the mean fuel mixture fraction $\tilde{Z}_{\text {fuel }}$ is not conserved. Tracing a conserved scalar is, however, a prerequisite for the mixing model outlined below. As previously mentioned, the conservation equation for the mean artifical scalar does not include any source terms (see equation (8)). Nevertheless, most of the fuel mixture formation process is already captured with the mean artificial scalar $\tilde{S}$, when mapping the distribution of the mean fuel mixture fraction $\tilde{Z}_{\text {fuel }}$ on it at the initialization point. This is due to the fact that the initialization of the interactive coupling usually takes place close to end of injection during PCCI combustion (see also section 3.2.1).

After initializing the multi-zone model, both codes interact back and forth throughout the engine cycle. The two codes run independently but simultaneously, without any reinitialization of the zones (and the numerical solver for the differential equations) in the multi-zone model.

Figure 1 schematically illustrates how the AC-FluX CFD code is used to initialize the zones in X0D, for which the chemistry is solved. For the reference case TS-3 (see sections 3.1 and 3.2), the left-hand side of Fig. 1 shows the injector cut plane coloured by zone affiliation at the time of XOD initialization. The zone affiliation is the result of the outlined sorting procedure. Upon initialization, the specific fuel masses, the mean temperatures, and the mean residual mass fractions of the zones in the physical

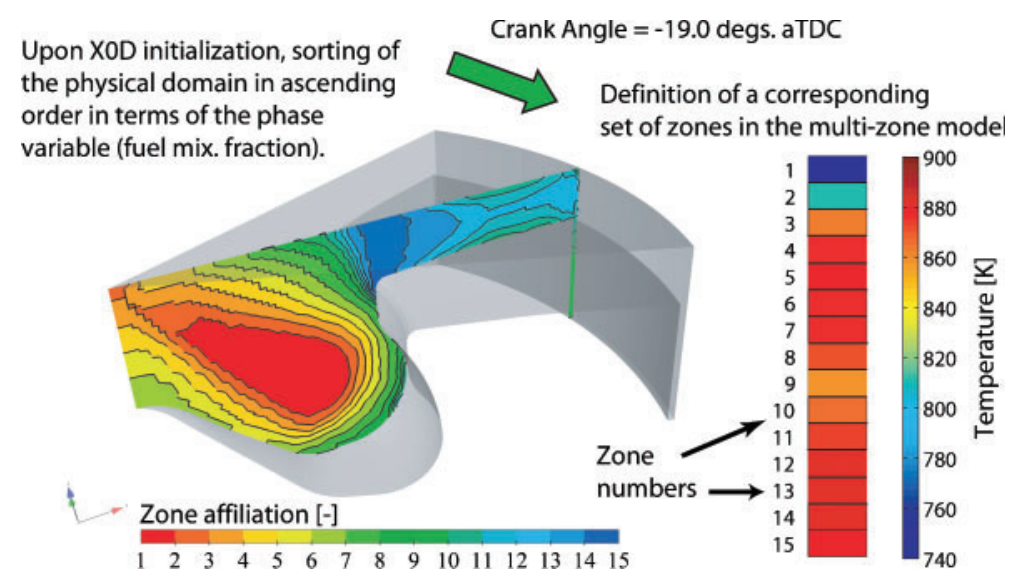

Fig. 1 Schematic illustration of the zone initialization in the multi-zone model 
domain are used to define a corresponding set of zones in the multi-zone model. This set of zones is shown in the right-hand side of Fig. 1. The zones are coloured according to their initial temperature.

\subsubsection{Mixing model}

In the engine, the mixing is controlled by the turbulence. Obviously, it is desirable to introduce mixing effects into the multi-zone model solving for the chemistry. This is done as follows. Assuming that, first, the combustion chamber is a closed system, meaning that the valves are closed; and second, the total mass is constant, then the distribution of the conserved scalars (e.g. the artificial scalar $\tilde{S}$ ) in the CFD code only changes due to convection and diffusion. The change for the mean artificial scalar $\tilde{S}_{i}$ from CFD time step $n-1$ to CFD time step $n$ in the individual zones can be described as

$$
m_{i} \times \frac{\tilde{S}_{i}^{n}-\tilde{S}_{i}^{n-1}}{\Delta t^{n}}=\sum_{k=1}^{2} \dot{m}_{i k}\left(\tilde{S}_{k}^{n-1}-\tilde{S}_{i}^{n-1}\right)
$$

where $\Delta t^{n}$ is the time increment, $m_{i}$ the mass of zone $i$, and $\dot{m}_{i k}$ the mass exchange rate of zone $i$ with neighbouring zone $k$ (see equations (1) and (2)). Since the phase space is one-dimensional, each zone can have up to two neighbours with which to exchange their scalar quantities.

Allowing mixing only between neighbouring zones is a logical choice, because transitions in phase space have to be continuous. If a state $B$ is intermediate between two states $\mathrm{A}$ and $\mathrm{C}$, then a zone of state A cannot transit to state $C$ without passing through state $\mathrm{B}$ during a diffusive process. The zones in phase space are therefore arranged in ascending (or descending) order, as illustrated in Fig. 1. Consequently, neighbouring zone mixing is used in the remainder of this paper. This is analogous to a RIF. In the flamelet equations, the scalar dissipation rate $\chi$ is an external parameter that is imposed on the flamelet structure. It can be thought of as a diffusivity in mixture fraction space that accounts for an exchange between adjacent reactive scalars (temperature and species mass fractions) in mixture fraction space. The laminar flamelet equations were derived by Peters $[\mathbf{3 6}, \mathbf{3 9}, \mathbf{4 0}]$ and integrated into the concept of RIFs by his co-workers [19, 41-43].

The mass exchange rate $\dot{m}_{i k}$ is expressed by the average of the neighbouring zones $i$ and $k$ according to

$$
\dot{m}_{i k}=0.5\left(\dot{m}_{i}+\dot{m}_{k}\right)
$$

In equation (10), $\dot{m}_{i}$ and $\dot{m}_{k}$ denote individual mass exchange rates of zones $i$ and $k$, respectively. Inserting equation (10) into equation (9) yields a system of $n z$ unknowns and $n z+1$ equations. The system is overdetermined, because there is no exchange over the boundaries of the phase space and, therefore, the summation of the exchange (see equation (9)) over all zones is zero. This constitutes an additional equation. The remedy is to simply fix the value for one of the individual mass exchange rates $\dot{m}_{i}$ (e.g. $\dot{m}_{1}=1$ ). The values for the mass exchange rates $\dot{m}_{i k}$ are defined by the two solutions for the mean artificial scalars $\tilde{S}_{i}$ at CFD time steps $n$ and $n-1$. No matter which value is assigned to one of the individual mass exchange rates $\dot{m}_{i}$, it does not change the resulting values for the mass exchange rates $\dot{m}_{i k}$.

\subsubsection{Chemical heat release}

The chemical source terms $\dot{\omega}_{j}$ (see equation (5)) couple the CFD code AC-FluX to the multi-zone model X0D and keep the heat release consistent between both codes. The fuel mass fraction $\tilde{Y}_{\text {fuel }}$ acts as a progress variable for the heat release. In the CFD code, the average rate of change for the fuel mass $\dot{m}_{\text {fuel, } i}$ in zone $i$ is coupled to the gross heat release in the multi-zone model through

$$
\dot{m}_{\mathrm{fuel}, i}=\frac{\dot{Q}_{\mathrm{ch}, \mathrm{XOD}, i}}{Q_{\mathrm{LHV}_{p}}}
$$

In equation (11), $\dot{Q}_{\mathrm{ch}, \mathrm{X0D}, i}$ denotes the gross heatrelease rate of zone $i$ that is taken from the multizone model. $Q_{\mathrm{LHV}_{p}}$ is the lower heating value of the fuel.

The actual value of the mean fuel mixture fraction in each CFD cell varies from the average value of the zone to which it belongs. For this reason, in order to properly distribute the conversion of fuel over the CFD cells, the average rate of change for the fuel mass $\dot{m}_{\text {fuel, } i}$ in zone $i$ is weighted with the ratio of the actual mean fuel mixture fraction in CFD cell ijk and the average mean fuel mixture fraction of the corresponding zone $i$. This yields

$$
\dot{m}_{\mathrm{fuel}, i j k}^{\mathrm{CFD}}=\frac{\tilde{Z}_{\mathrm{fuel}, i j k}^{\mathrm{CFD}}}{\tilde{Z}_{\mathrm{fuel}, i}} \times \dot{m}_{\mathrm{fuel}, i}
$$

where $\dot{m}_{\mathrm{fuel}, i j k}^{\mathrm{CFD}}$ denotes the rate of change for the fuel mass in CFD cell $i j k$ and $\tilde{Z}_{\mathrm{fuel}, i j k}^{\mathrm{CFD}}$ the actual mean fuel mixture fraction in CFD cell $i j k$. 
The other species (air and products of combustion) which are accounted for in AC-FluX are computed via a one-step global reaction corresponding to the stoichiometry.

\subsubsection{Source terms}

The conservation equation for the mean total enthalpy $\tilde{h}$ can be written as

$$
\frac{\partial \bar{\rho} \tilde{h}}{\partial t}+\frac{\partial \bar{\rho} \tilde{v}_{i} \tilde{h}}{\partial x_{i}}=\frac{\partial}{\partial x_{i}}\left(\bar{\rho} D_{h} \frac{\partial \tilde{h}}{\partial x_{i}}\right)+\frac{D \bar{\rho}}{D t}+\dot{q}^{\mathrm{s}}-\dot{q}_{\text {wall }}
$$

It has two source terms $\left(\dot{q}^{\mathrm{s}}\right.$ and $\left.\dot{q}_{\text {wall }}\right)$ that need to be accounted for in the multi-zone model X0D.

Vaporization of fuel in the multi-zone model is accounted for by averaging the source term $\dot{\rho}_{\text {fuel }}^{\mathrm{s}}$ in equation (7) for each zone in the CFD code. This averaging procedure yields the source term $\dot{\rho}_{i j, j=\text { fuel }}^{\mathrm{s}}$ in equations (1) and (2) and is linked to the source term for the spray in equation (13) via

$$
\dot{q}_{i}^{\mathrm{s}}=\sum_{j=1}^{n s p} \dot{\rho}_{i j}^{\mathrm{s}} \times \Delta h_{v j}, \quad \text { with } \quad \dot{\rho}_{i j}^{\mathrm{s}}=0, j \neq \text { fuel }
$$

In X0D, wall heat transfer (see equations (2) and (4)) is described as

$$
\dot{q}_{\text {wall }, i} \times V_{i}=\dot{Q}_{\text {wall }, i}=\sum_{l=1}^{n_{\text {wall }}} A_{\text {wall }, l, i} \times h_{\text {wall }, l, i}\left(T_{i}-T_{\text {wall }, l}\right)
$$

The area $A_{\text {wall, } l, i}$ of wall $l$ belonging to zone $i$ is taken from the CFD solution and updated in the multizone model at every CFD time step. The heat transfer coefficient $h_{\text {wall, } l, i}$ of zone $i$ to wall $l$ is continuously recalculated based on the CFD solution and updated in the multi-zone model at every CFD time step such that the wall heat transfer in the multi-zone model exactly matches the one in the CFD code. The recalculation of $h_{\text {wall } l, i}$ uses the source term $\dot{q}_{\text {wall }}$ in equation (13), also averaged for each zone in the CFD code.

The pressure change in $\mathrm{XOD}$ is described by equation (3), where the spatial pressure fluctuations are neglected and only the average cylinder pressure change, which is due to the volume change and heat release, is taken into account. The volume change in both codes is computed by using the same slidercrank-equation [31].
Thereby, the full interactive coupling of AC-FluX and $\mathrm{X} 0 \mathrm{D}$ is accomplished.

\subsection{Systematic reduction of the interactively coupled CFD-multi-zone approach towards a stand-alone multi-zone model}

Section 2.3 depicts the exchange of information between the CFD code and the multi-zone model. Two information streams and an appropriate treatment of the source terms establish the interactive coupling of both codes. This subsection presents a methodology of how the information that is fed from the CFD code to the multi-zone model can be accounted for within the multi-zone model. This allows for decoupling the multi-zone model from the CFD code and leads to a systematic reduction of the interactively coupled CFD-multi-zone approach towards a stand-alone multi-zone model. Sections 2.4.1 and 2.4.2 describe an appropriate modelling of the sink and source terms (heat transfer to the walls, fuel injection, and vaporization) within the multi-zone model. Finally, section 2.4 .3 presents a simplified mixing model within the multi-zone model.

\subsubsection{Heat transfer to the walls}

In the multi-zone model, heat transfer to the walls is accounted for by equation (4). Instead of using CFD information to calculate the heat transfer coefficient $h_{\text {wall } l, i}$, it can also be modelled with the Woschni correlation [44], which is

$$
\begin{aligned}
h_{\mathrm{wall}, l, i} & =h_{\mathrm{wall}} \\
& =3.26 \times b^{-0.2} \times p^{0.8} \times T_{\mathrm{av} .}^{-0.53}\left(C_{1} \times v_{\text {piston }}\right)^{0.8}
\end{aligned}
$$

where $b$ is the bore of the engine, $p$ the mean cylinder pressure, $T_{\mathrm{av}}$. the average in-cylinder temperature, and $v_{\text {piston }}$ the mean piston speed. The constant $C_{1}$ is adjustable and is set to 0.5 . Since the average in-cylinder temperature $T_{\mathrm{av}}$. is used within the Woschni correlation and $h_{\text {wall }, l, i}$ does not depend on any specific wall, the heat transfer coefficient is equal for all zones and walls, leading to $h_{\mathrm{wall}, l, i}=$ $h_{\text {wall. }}$.

For the specific engine investigated, the area $A_{\text {wall, } l, i}$ of wall $l$ belonging to zone $i$ has to be taken from a preceding simulation with the interactively coupled CFD-multi-zone approach so that this 
information can be incorporated into a successive simulation with the stand-alone multi-zone model.

\subsubsection{Fuel injection and evaporation}

A preceding simulation with the interactively coupled CFD-multi-zone approach also allows the determination of the amount of fuel that is injected and vaporizes in each CFD zone. This information can then be incorporated into a successive simulation with the stand-alone multi-zone model (see source term $\dot{\rho}_{i j, j=\text { fuel }}^{\mathrm{s}}$ in equations (1) and (2)).

\subsubsection{Simplified mixing model}

The simplified mixing model within the stand-alone multi-zone model assumes that the previously introduced mass exchange rate $\dot{m}_{i k}$ of zone $i$ with neighbouring zone $k$ (see equations (1) and (2)) is proportional to the wall heat transfer coefficient $h_{\text {wall }}$ and the area $A$ both zones share [27]. This is an analogy to the well-known empirical Lewis' law which assumes a similarity between heat and mass transfer [45]. The heat transfer to the walls and the mass exchange between the zones are both induced by the turbulence intensity so that they can be related to each other. The corresponding equation is

$$
\dot{m}_{i k}=r_{\mathrm{MB}} \times A \times \frac{m_{i}+m_{k}}{\left(m_{i} \bar{c}_{\mathrm{p} i}+m_{k} \bar{c}_{\mathrm{p} k}\right)} \times h_{\mathrm{wall}}
$$

In equation (17), $\bar{c}_{\mathrm{p} i}$ is the average heat capacity at constant pressure of zone $i . r_{\mathrm{MB}}$ is a parameter that is adjusted to yield the desired level of mixing. The exact choice of $r_{\mathrm{MB}}$ is discussed in section 4.2.1 below. The area $A$ is computed from the volumes $V_{i}$ and $V_{k}$ of the two zones $i$ and $k$ according to

$$
A=4 \pi\left[\frac{3}{4 \pi}\left(V_{i}+V_{k}\right)\right]^{2 / 3}
$$

\subsection{Computational accuracy and efficiency}

\subsubsection{Interactively coupled CFD-multi-zone approach}

The interactively coupled CFD-multi-zone approach efficiently separates the solution of the flow field from the solution of the chemistry. During one time step of the CFD code, the multi-zone code solves equations (1), (2), and (3) with time steps that can be much smaller, depending on the chemistry. In this way, the time scales of the chemistry and the fluid dynamics are decoupled. Thus, the approach does not need to simplify the highly non-linear chemistry, allowing for any detailed reaction mechanisms to be used. These mechanisms may also include pollutant formation and destruction. This procedure yields an approach that includes an accurate description of PCCI chemistry with only a small penalty in computational cost, since even during auto-ignition, the CFD time step has hardly to be reduced compared to an inert computation of the chemistry.

Decoupling the time scales of the chemistry and the fluid dynamics proved successful in predicting conventional diesel engine combustion in the framework of RIFs.

\subsubsection{Stand-alone multi-zone model}

In comparison to the interactively coupled CFDmulti-zone approach, the stand-alone multi-zone model does not solve for the solution of the flow field. This yields a significant advantage in computational costs. However, modelling the CFD information according to the procedure outlined in section 2.4 reduces the accuracy of the simulation outcome. This reduction of accuracy is discussed further in sections 4.2.1 and 4.2.2.

\subsubsection{Run time comparison}

The computations with both the interactively coupled CFD-multi-zone approach and the standalone multi-zone model were carried out on a Dell PowerEdge 1950 with two Intel Xeon 5160 CPUs (dual core with $3.0 \mathrm{GHz}$ ). For this, both approaches written in FORTRAN 77 were compiled with the Intel Fortran Compiler 9.1.043. They were executed for one high-pressure engine cycle. Sections 3.2.1 and 3.2.2 provide a detailed description of the numerical setups. Table 1 contains a run time comparison for both approaches, depending on the number of zones or the number of partial differential equations, respectively. Thirteen partial differential equations are solved in the CFD code (mass, momentum, total enthalpy, active species stream, mean fuel mixture fraction, and artificial scalar conservation, k-epsilonequations; see section 2.2). The equations in the CFD code are evaluated on each grid cell. In the multizone model, 40 partial differential equations (species mass fractions, temperature, and pressure change; see sections 2.1.2 and 2.1.3) are solved per zone.

For the interactively coupled CFD-multi-zone approach, the CPU time costs are approximately 
Table 1 Run time comparison for the interactively coupled CFD-multi-zone approach and the stand-alone multizone model. Depending on the number of zones, the left column contains the number of partial differential equations that are solved in the CFD-multi-zone approach or the stand-alone multi-zone model, respectively. The second and third columns contain the corresponding CPU times for one high-pressure engine cycle simulation

\begin{tabular}{lll}
\hline $\begin{array}{l}\text { Number of zones/number of equations for CFD-multi-zone } \\
\text { approach or stand-alone multi-zone model }\end{array}$ & $\begin{array}{l}\text { CPU time for interactively coupled } \\
\text { CFD-multi-zone approach (hours) }\end{array}$ & $\begin{array}{l}\text { CPU time for stand-alone } \\
\text { multi-zone model (minutes) }\end{array}$ \\
\hline 5 zones/13+200 equations or 200 equations, respectively & $\sim 24.1$ & $\sim 0.2$ \\
10 zones/13+400 equations or 400 equations, respectively & $\sim 24.8$ & $\sim 1.1$ \\
15 zones/13+600 equations or 600 equations, respectively & $\sim 25.5$ \\
20 zones/13+800 equations or 800 equations, respectively & $\sim 28.0$ \\
25 zones/13+1000 equations or 1000 equations, respectively & $\sim 36.0$ & $\sim 3.3$ \\
\hline
\end{tabular}

$24 \mathrm{~h}$ for the 13 differential equations solved in the CFD code. The CPU time for the multi-zone model depends on the total number of zones, varying between less than $10 \mathrm{~min}$ and $12 \mathrm{~h}$. It roughly scales with the number of equations cubed, indicating that the stiffness of the Jacobian in the multi-zone model is nearly independent of the number of zones.

It can be seen from Table 1 that the stand-alone multi-zone model is significantly faster in terms of computational time than the interactively coupled CFD-multi-zone approach. This is due to the fact that the mass exchange rates $\dot{m}_{i k}$ computed within the stand-alone multi-zone model have a smoother progression throughout the simulations in comparison to the interactively coupled CFD-multi-zone approach.

\section{EXPERIMENTAL AND NUMERICAL SETUP}

\subsection{Experimental setup}

\subsubsection{Engine}

At the Institut für Technische Verbrennung at RWTH Aachen University, Germany, experiments were carried out with a 1.91 GM Fiat diesel engine. This engine is equipped with a second-generation Bosch common-rail injection system. All relevant engine data are provided in Table 2. The mounting of the engine on the test bench is shown in Fig. 2. A more detailed description regarding the engine, the test cell equipment, and the injection rate measurements can be found in Vanegas et al. [46].

\subsubsection{Operating conditions}

The engine operating conditions are summarized in Table 3. The engine was operated at part-load conditions with a speed of $2000 \mathrm{r} / \mathrm{min}$ and an external EGR rate of 15,30 , or 45 per cent, respectively. The external EGR was cooled to about
Table 2 GM Fiat engine specifications

\begin{tabular}{ll}
\hline Description & Value \\
\hline Displacement volume & $1910 \mathrm{~cm}^{3}$ \\
Bore & $82.0 \mathrm{~mm}$ \\
Stroke & $90.4 \mathrm{~mm}$ \\
Bore distance & $90.0 \mathrm{~mm}$ \\
Cylinder block height & $236.5 \mathrm{~mm}$ \\
Connecting rod length & $145.0 \mathrm{~mm}$ \\
Compression height & $46.5 \mathrm{~mm}$ \\
Valve diameter $I / E$ & $28.5 / 25.5 \mathrm{~mm}$ \\
Compression ratio & $17.5: 1$ \\
Output at engine speed & $110 \mathrm{~kW} / 4000 \mathrm{r} / \mathrm{min}$ \\
Torque at engine speed & $315 \mathrm{~kW} / 2000 \mathrm{r} / \mathrm{min}$ \\
Idle speed & $850 \mathrm{r} / \mathrm{min}$ \\
Maximum speed & $5100 \mathrm{r} / \mathrm{min}$ \\
Injection system & $7-\mathrm{hole} \mathrm{injector}$ \\
Included spray angle & $100.0^{\circ}$ \\
Hole diameter & $0.141 \mathrm{~mm}$ \\
Flow number & $440 \mathrm{~cm} / 30 \mathrm{~s}$ at $100 \mathrm{bar}$ \\
Swirl number & 2.5 \\
Fuel & diesel \\
\hline
\end{tabular}

$350 \mathrm{~K}$ before mixing with the fresh air in the intake section. Ten variations of SOI were investigated for each external EGR rate, leading to 30 different experiments. The SOI variation started at $-45.7^{\circ}$ crank angle after top dead centre (CA aTDC) and lasted until $-0.7^{\circ} \mathrm{CA}$ aTDC, with a time shift of $5.0^{\circ}$ CA between two consecutive SOIs. For all these experiments, the total fuel mass injected was $10.2 \mathrm{mg} / \mathrm{cycle}$, with an injection duration of $8.8^{\circ}$ CA. Two additional SOI variations were carried out with an external EGR rate of 30 per cent and a reduced total fuel mass injected of $6.8 \mathrm{mg} / \mathrm{cycle}$, or an increased total fuel mass injected of $13.6 \mathrm{mg} /$ cycle, respectively, leading to another 20 different experiments. In these additional experiments, the injection duration was $5.9^{\circ}$ or $11.7^{\circ} \mathrm{CA}$, depending on the total fuel mass injected. The rail pressure was 700 bar for all 50 different engine operating conditions.

In section 4 , the interactively coupled CFD-multizone approach, as well as its reduction to the standalone multi-zone model, is exemplarily validated against five selected operating conditions. This 


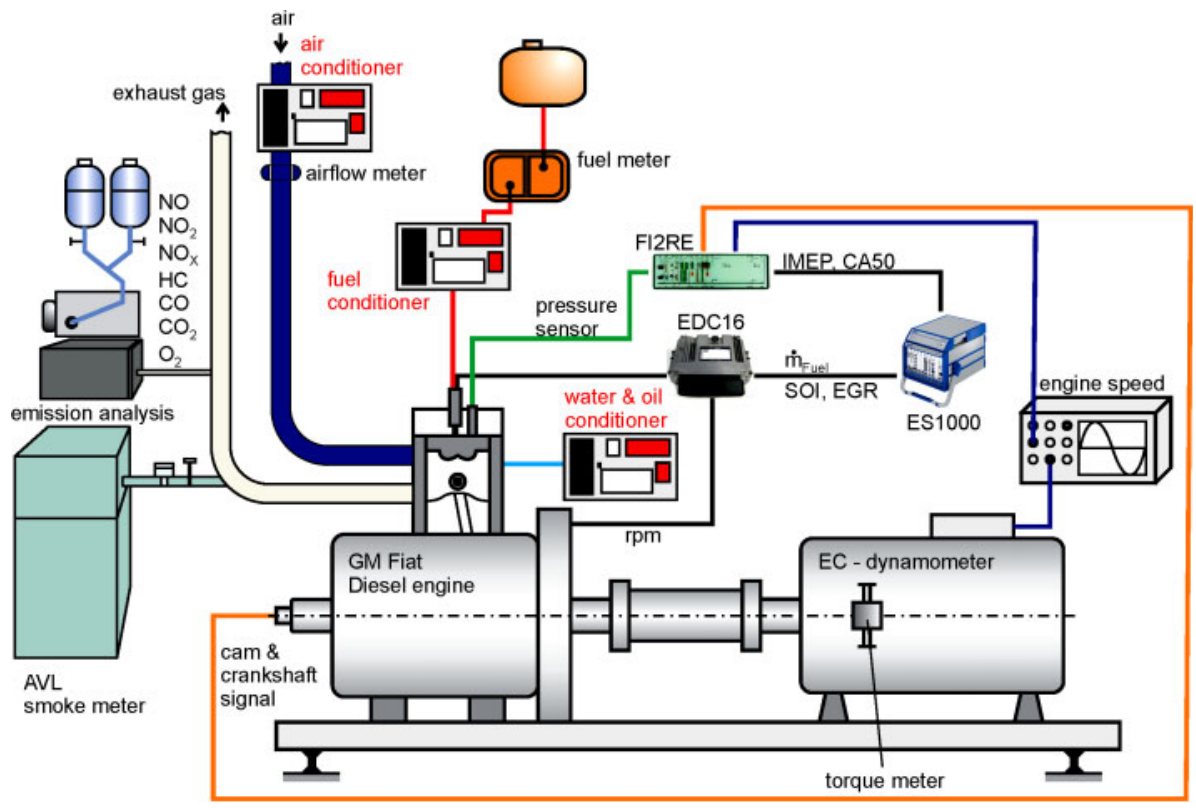

Fig. 2 Engine test bench

Table 3 Engine operating conditions

\begin{tabular}{ll}
\hline Description & Value \\
\hline Engine speed & $2000 \mathrm{r} / \mathrm{min}$ \\
Rail pressure & $700 \mathrm{bar}$ \\
Fuel mass injected & $6.8,10.2$, or $13.6 \mathrm{mg} / \mathrm{cycle}$ \\
Relative air/fuel ratio & between 5.3 and 1.6 \\
External EGR rate & 15,30, or $45 \%$ \\
SOI & $-45.7^{\circ} \mathrm{CA}$ aTDC \\
& $-40.7^{\circ} \mathrm{CA}$ aTDC \\
& $-35.7^{\circ} \mathrm{CA}$ aTDC \\
& $-30.7^{\circ} \mathrm{CA}$ aTDC \\
& $-25.7^{\circ} \mathrm{CA}$ aTDC \\
& $-20.7^{\circ} \mathrm{CA}$ aTDC \\
& $-15.7^{\circ} \mathrm{CA}$ aTDC \\
& $-10.7^{\circ} \mathrm{CA}$ aTDC \\
& $-5.7^{\circ} \mathrm{CA}$ aTDC \\
& $-0.7^{\circ} \mathrm{CA}$ aTDC \\
\hline
\end{tabular}

includes five variations of SOI $\left(-35.7^{\circ},-30.7^{\circ}\right.$, $-25.7^{\circ},-20.7^{\circ}$, and $-15.7^{\circ} \mathrm{CA}$ aTDC) at an external EGR rate of 30 per cent which are referred to as timings $1,2,3,4$, and 5 (TS-1, TS-2, TS-3, TS-4, and TS-5) in the remainder of the paper.

\subsection{Numerical setup}

\subsubsection{Interactively coupled CFD-multi-zone approach}

Computations with the interactively coupled CFDmulti-zone approach were performed for all engine operating conditions presented in section 3.1.2. All computations started from intake valve closure (IVC) at $-165.6^{\circ} \mathrm{CA}$ aTDC and ended at exhaust valve opening (EVO) at $149.1^{\circ} \mathrm{CA}$ aTDC. In the first part of the CFD simulation, AC-FluX was run alone. For every operating condition, the coupling to X0D was initialized $7.0^{\circ} \mathrm{CA}$ after start of injection. The starting solution at IVC was initialized with pressure and temperature taken from the experiments. The internal EGR rate was assumed to be 5 per cent. The velocity field was initialized with a swirl number of 2.5 .

Two different computational meshes were used throughout the simulations to provide an optimal computational mesh for any piston position. One grid was used for the compression phase and one for the combustion and expansion phase. This procedure allowed for rearranging the cells shortly before the beginning of the combustion event. In the computational mesh for the combustion and expansion phase, in comparison to the one for the compression phase, the mesh resolution in the bowl region was refined. For both meshes, a cell layer removal technique was applied in the cylinder region throughout the simulations. This was done in order to account for the compression and expansion of the grid cells along the cylinder axis due to the piston movement. The remap between both grids took place at $-37.0^{\circ} \mathrm{CA}$ aTDC. There is no sensitivity on the results when advancing or retarding the remap of the solution.

An outline of the computational mesh for the combustion phase is given in Fig. 3. The simulation used a sector grid representing one-seventh of the combustion chamber, thereby taking advantage of the axial symmetry with respect to the placement of 


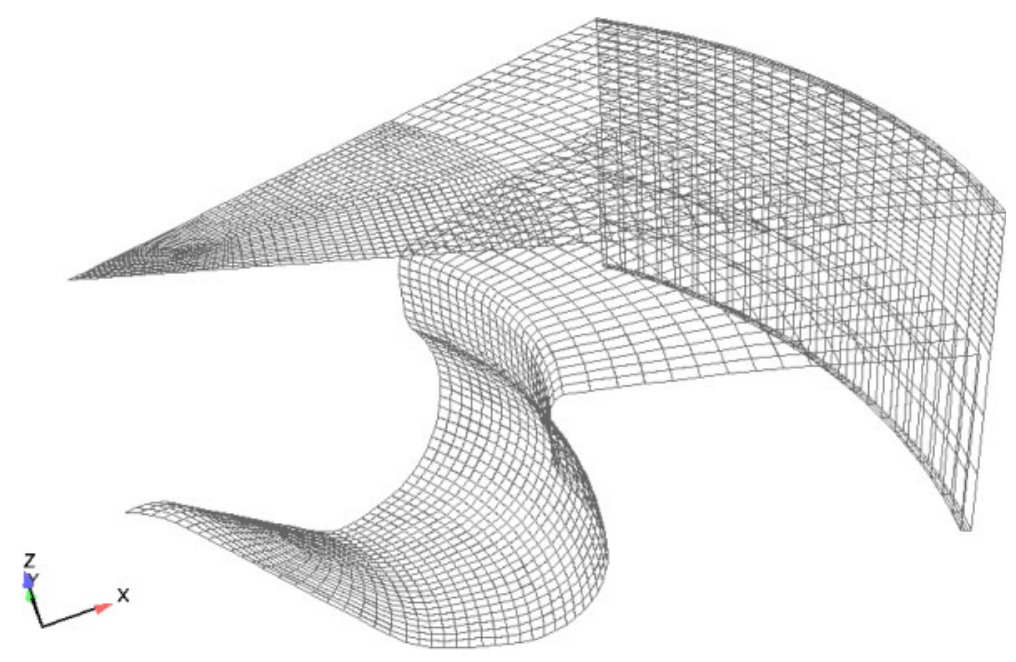

Fig. 3 Computational grid at $-25.0^{\circ} \mathrm{CA}$ aTDC. Cyclic boundaries are removed for illustration purposes

the nozzle holes. In Fig. 3, the cyclic boundaries are removed for the sake of a clear insight into the bowl. The mesh size was 52704 cells at TDC. A sensitivity study revealed that this was a sufficient resolution for all cases.

The wall temperatures were set based on experimental experience and held constant during the simulations. The spray model parameters are always subject to adjustment and were adapted such that the experimental and simulated pressure curve for TS-3 matched. Otherwise they were held unchanged for all additional simulations. The parameters used in this work are within the recommended parameter range proposed by Weber et al. [47].

\subsubsection{Stand-alone multi-zone model}

For all engine operating conditions presented in section 3.1.2, computations were also performed with the stand-alone multi-zone model. For reasons of consistency, the initial conditions, start and end of simulation, number of zones, etc., were held identical to the simulations carried out with the interactively coupled CFD-multi-zone approach.

TS-3 was used as a calibration case for incorporating information from the interactively coupled CFD-multi-zone approach into the stand-alone multi-zone model ( $\dot{\rho}_{i j}^{\mathrm{s}}$ and $A_{\text {wall, }, i}$, , see equations (1), (2), and (4)). The information obtained for TS-3 from the simulation with the interactively coupled CFD-multi-zone approach was used for all experiments investigated with the stand-alone multi-zone model. This introduces a small error into the other stand-alone multi-zone model simulations (e.g. TS1, TS-2, TS-4, and TS-5). However, this error is small, since the calibration conditions of TS-3 do not deviate significantly from all other engine operating conditions.

\section{STATIONARY VALIDATION}

In this section, the interactively coupled CFD-multizone approach is first validated against experimental data in terms of pressure curves, $\mathrm{IMEP}_{\mathrm{HP}}, \mathrm{CA10}$, CA50, CO emissions, combustion efficiency, and $\mathrm{NO} / \mathrm{NO}_{x}$ emissions for all five aforementioned SOI timings (TS-1, TS-2, TS-3, TS-4, and TS-5). After this, TS-3 is considered in detail. The exchange of information between the CFD code and the multizone model within the interactively coupled CFDmulti-zone approach is investigated. Following this, the systematic reduction of the interactively coupled CFD-multi-zone approach towards the stand-alone multi-zone model is exemplarily shown for TS-3. Finally, simulation results obtained with the standalone multi-zone model for all five SOI timings are compared to experimental data.

\subsection{Interactively coupled CFD-multi-zone approach}

\subsubsection{Sensitivity study}

A sensitivity study of the local in-cylinder quantities (e.g. temperature, fuel mass fraction), the ignition timing, and the overall heat-release rate on the number of zones was performed first. Beyond $n z=15$, the change of the local in-cylinder quantities, the ignition timing, and the overall heat- 

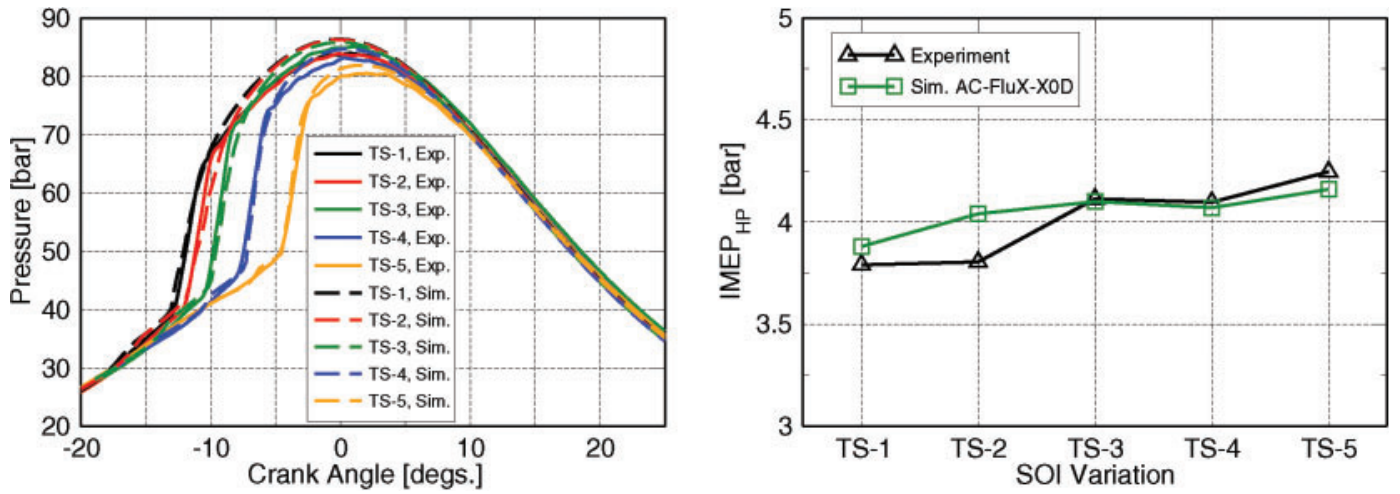

Fig. 4 Average cylinder pressure (left) and $\mathrm{IMEP}_{\mathrm{HP}}$ (right) for the SOI variation. Comparison between simulation with the interactively coupled CFD-multi-zone approach and experiment

release rate was insignificant, and thus a number of zones of $n z=15$ was chosen for all subsequent simulations.

\subsubsection{SOI variation}

Figure 4 compares the pressure curves and the corresponding IMEPs for the high-pressure part of the engine cycle $\left(\mathrm{IMEP}_{\mathrm{HP}}\right)$ obtained from the

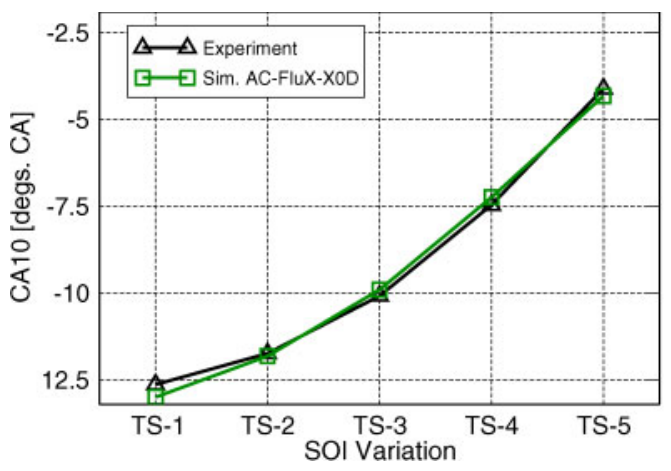

simulations with the interactively coupled CFDmulti-zone approach with the results from the experiments for the complete SOI variation TS- 1 to TS-5. It becomes evident that with retarded SOI timings the maximum pressure peak decreases, while the $\mathrm{IMEP}_{\mathrm{HP}}$ increases. For all five cases, the simulations are in good agreement with the experiments. There is only a slight overestimation of the maximum peak pressure in all simulations. The

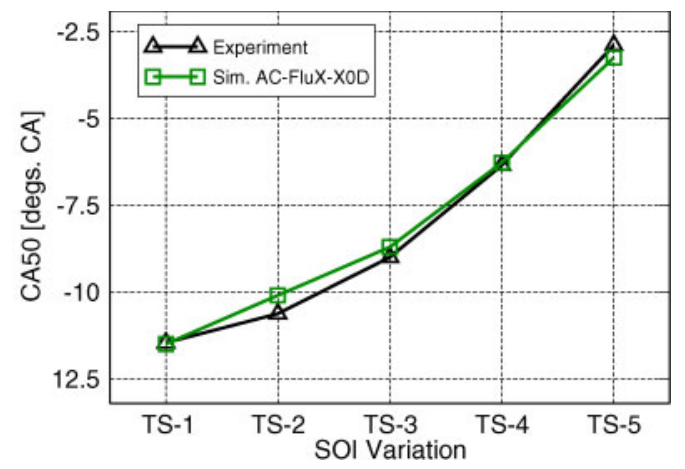

Fig. 5 CA10 (left) and CA50 (right) for the SOI variation. Comparison between simulation with the interactively coupled CFD-multi-zone approach and experiment
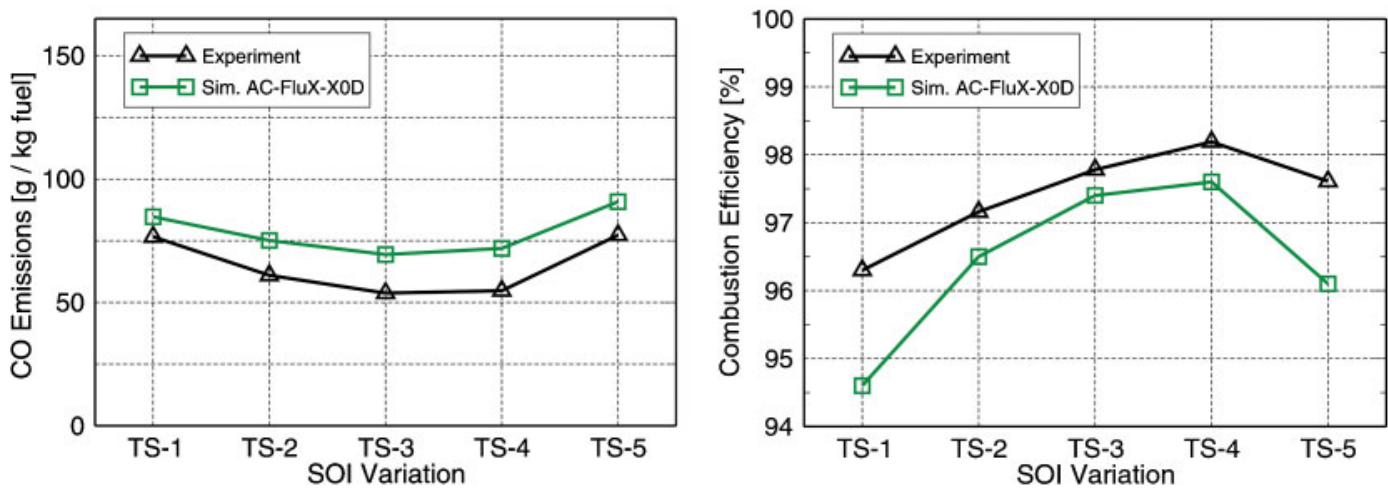

Fig. $6 \mathrm{CO}$ emissions in grammes/kilogramme fuel in the exhaust gas (left) and combustion efficiency in per cent (right) for the SOI variation. Comparison between simulation with the interactively coupled CFD-multi-zone approach and experiment 
qualitative trends are reproduced well by the simulations.

Figure 5 shows the crank angles at which 10 per cent (CA10) and 50 per cent (CA50) of the total injected fuel mass is burned for all simulations and experiments. Whereas CA10 is predicted almost perfectly, the simulated CA50 has a very slight but acceptable deviation from some of the experimental results.

The CO emissions in grammes/kilogramme fuel in the exhaust gas and the combustion efficiency in per cent for the complete SOI variation are summarized in Fig. 6. The CO emissions, which are rather challenging to predict, are slightly overestimated in the simulations. This leads to an underestimation of the combustion efficiency, since the simulated hydrocarbon (HC) emissions (not shown here) are roughly of the same order of magnitude as the experimentally obtained ones for all cases. All in all, the qualitative and quantitative agreement over the whole SOI variation is reasonable for both CO emissions and combustion efficiency.

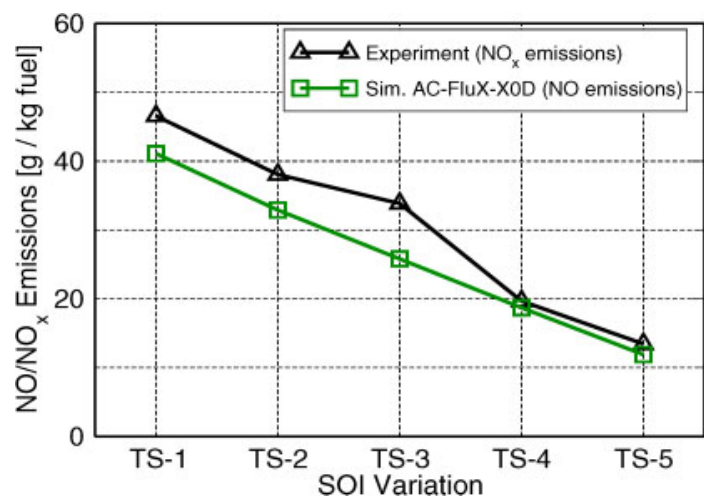

Fig. $7 \mathrm{NO} / \mathrm{NO}_{x}$ emissions in grammes/kilogramme fuel in the exhaust gas for the SOI variation. Comparison between simulation with the interactively coupled CFD-multi-zone approach and experiment

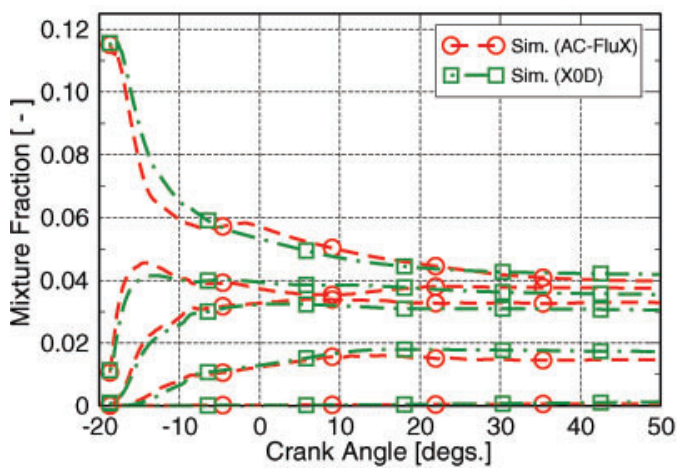

Finally, Fig. 7 presents a comparison between numerically and experimentally obtained $\mathrm{NO} / \mathrm{NO}_{x}$ emissions in grammes/kilogramme fuel in the exhaust gas for the complete SOI variation. There is good agreement. Note that the chemical mechanism used in the simulations only accounts for thermal NO (see also section 2.1.3), while both NO and $\mathrm{NO}_{2}$ emissions are measured in the experiments. $\mathrm{NO}$ and $\mathrm{NO}_{2}$ emissions are usually grouped together as $\mathrm{NO}_{x}$ emissions, with $\mathrm{NO}$ being the predominant oxide of nitrogen produced inside the engine cylinder [31].

\subsubsection{Exchange of information between the CFD code and the multi-zone model for TS-3}

For TS-3, Fig. 8 shows the fuel mixture fraction and the temperature distribution in five representative AC-FluX zones and in their corresponding X0D zones around TDC firing. At time of X0D initialization $\left(-18.7^{\circ} \mathrm{CA}\right.$ aTDC), the mean fuel mixture fractions $\tilde{Z}_{\text {fuel, } i}$ vary roughly between 0.0 and 0.125 . With 30 per cent external and 5 per cent internal EGR, the stoichiometric mixture fraction of diesel fuel is approximately $Z_{\text {diesel, st }} \approx 0.053$. Thus, some in-cylinder zones are fuel-rich, whereas most others are fuel-lean. Throughout the simulation, the mean fuel mixture fraction stratification decreases due to mixing and diffusion processes. The evolution of the fuel mixture fractions in $\mathrm{X} 0 \mathrm{D}$ is almost identical to that of AC-FluX. The mixing model accurately replicates the mixing between the zones in phase space and keeps the mixing and distribution of the phase variable consistent between the CFD code and the multi-zone model at all times. Hence, mixing and diffusion processes are captured well in X0D. The evolution of the temperature in AC-FluX and in X0D for the same five representative zones also matches well. The exchange of information between AC-FluX and X0D as outlined in section 2.3 keeps the

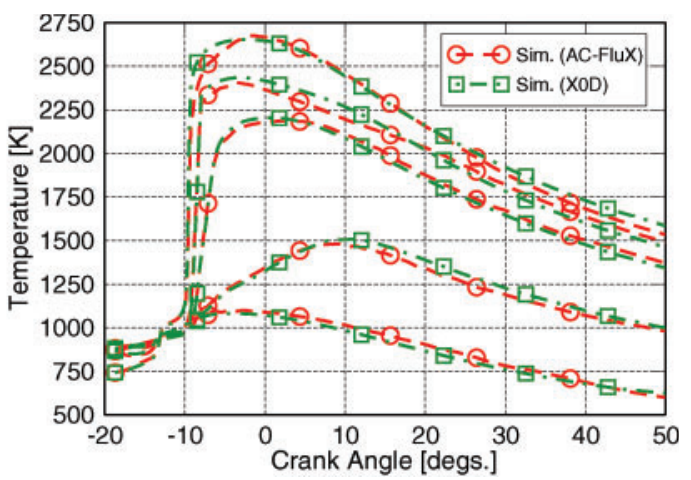

Fig. 8 Comparison of fuel mixture fraction (left) and temperature (right) for XOD and AC-FluX for five selected zones for TS-3 

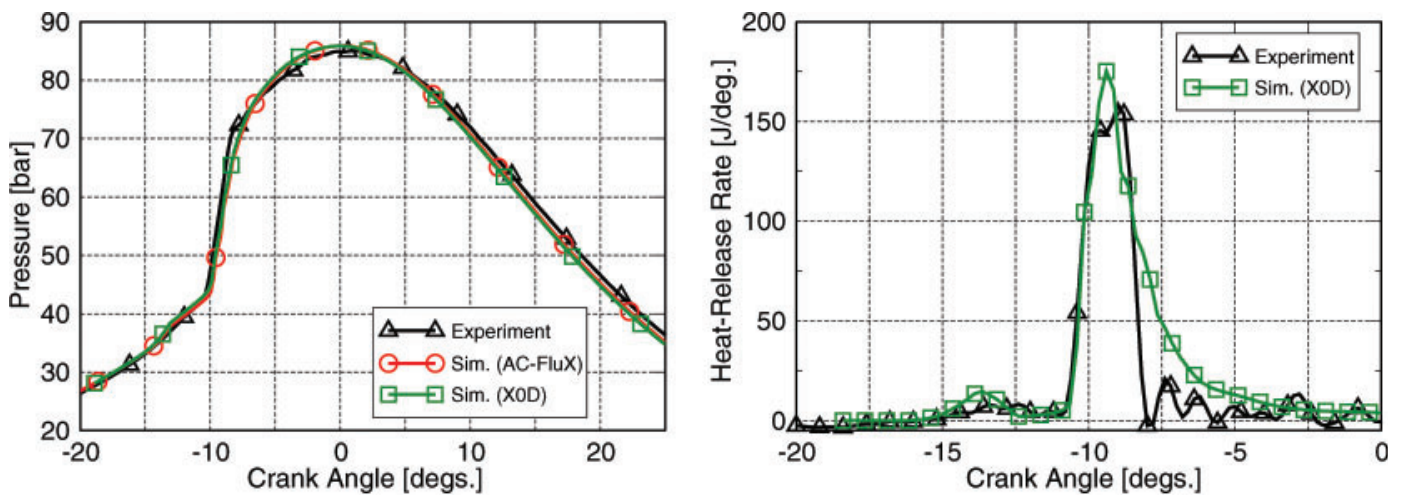

Fig. 9 Average cylinder pressure (left) and average apparent net heat-release rates (right) for TS3. Comparison between simulation and experiment. Simulation results are extracted from the CFD code and/or the multi-zone model

temperature evolution in both codes identical. Only small differences exist. However, these deviations are in an acceptable range. This validates the interactive coupling of both codes. For TS-3, the zones with the highest peak temperatures are also the ones with the highest fuel mixture fractions. Figure 8 shows that these zones auto-ignite first. Subsequently, the other zones auto-ignite or are ignited through heat and mass transfer from other zones, respectively. The coldest zone contains the least amount of fuel and has the smallest increase in temperature. The left-hand side of Fig. 9 compares the pressure trace obtained from the experiment with the ones from AC-FluX and X0D. The pressure traces of AC-FluX and X0D are in perfect agreement over the whole CA range, especially during the heatrelease phase. Only negligible differences exist. The simulated pressure curves reveal that auto-ignition is captured well in the simulation. The peak pressures match, too. The right-hand side of Fig. 9 displays the

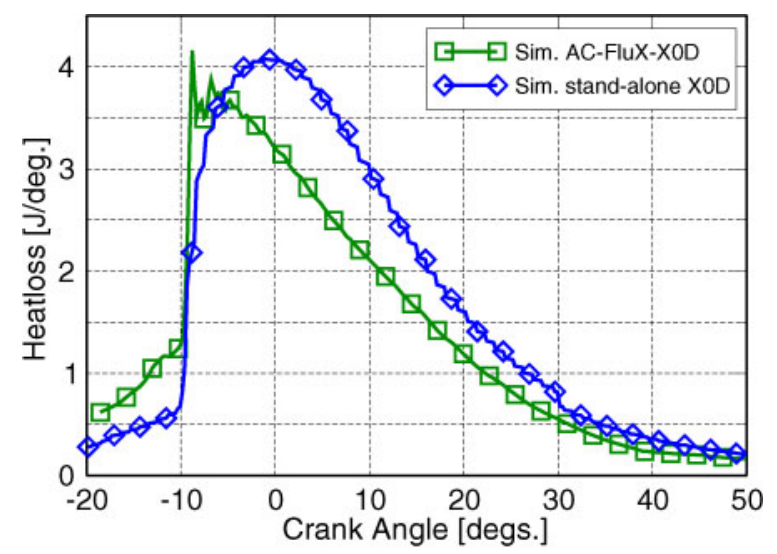

Fig. 10 Comparison of the integral heat transfer to all cylinder walls for the interactively coupled CFD-multi-zone approach and the standalone multi-zone model for TS-3 corresponding average apparent net heat-release rates. The comparison of the apparent net heatrelease rates further shows that first and second stage ignition, as well as the subsequent heat release, are reproduced well by the simulation. Only small discrepancies with respect to overall burning time exist. The CA50 obtained from the experiment is $-9.0^{\circ}$ CA aTDC. The simulation yielded a CA50 of $-8.7^{\circ} \mathrm{CA}$ aTDC (see also Fig. 5).

\subsection{Stand-alone multi-zone model}

\subsubsection{Systematic reduction for TS-3}

Sections 2.4.1, 2.4.2, and 2.4.3 outline how to systematically model CFD information within the multi-zone model. Among them, section 2.4.1 deals with the treatment of heat transfer to the walls. For

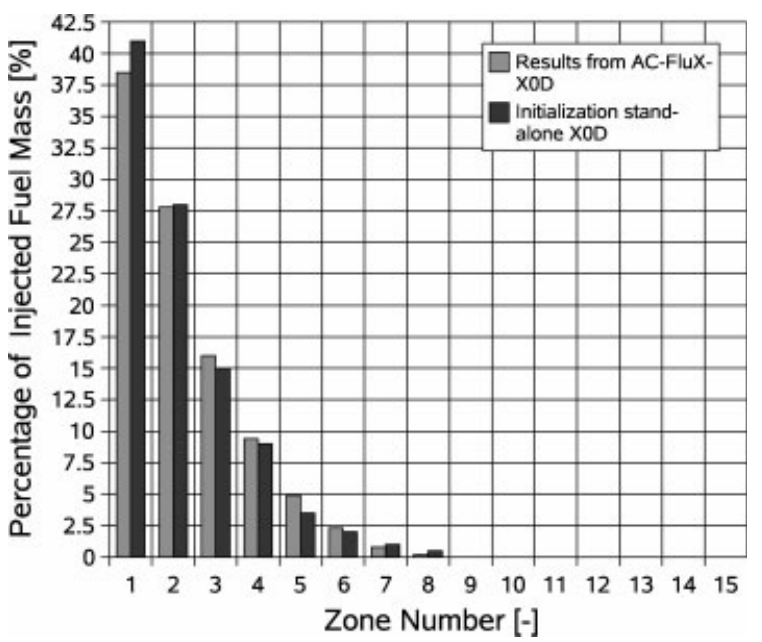

Fig. 11 Distribution of the vaporized fuel over all zones as obtained from the interactively coupled CFD-multi-zone approach and as incorporated into the stand-alone multi-zone model for TS-3 


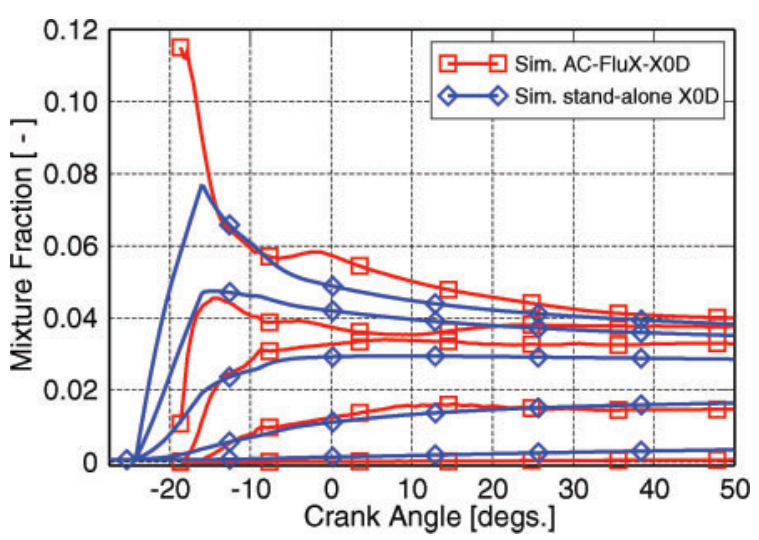

Fig. 12 Comparison of fuel mixture fraction for the interactively coupled CFD-multi-zone approach and the stand-alone multi-zone model for five selected zones for TS-3

TS-3, Fig. 10 presents a comparison of the integral heat transfer to all cylinder walls for the interactively coupled CFD-multi-zone approach and the standalone multi-zone model. Both are in good agreement.

Sections 2.4.2 and 2.4.3 deal with the fuel injection and vaporization, as well as with a simplified mixing model, within the stand-alone multi-zone model. In Fig. 11, the distribution of the vaporized fuel over the zones as obtained from the simulation with the interactively coupled CFD-multi-zone approach is shown for TS-3. Figure 11 also shows the distribution of the vaporized fuel over the zones as incorporated into the corresponding simulation with the stand-alone multi-zone model. Both are almost identical. In this context, Fig. 12 presents the fuel mixture fraction distribution for the interactively coupled CFD-multi-zone approach and the standalone multi-zone model for the same five representative zones already shown in Fig. 8. The incorpora-

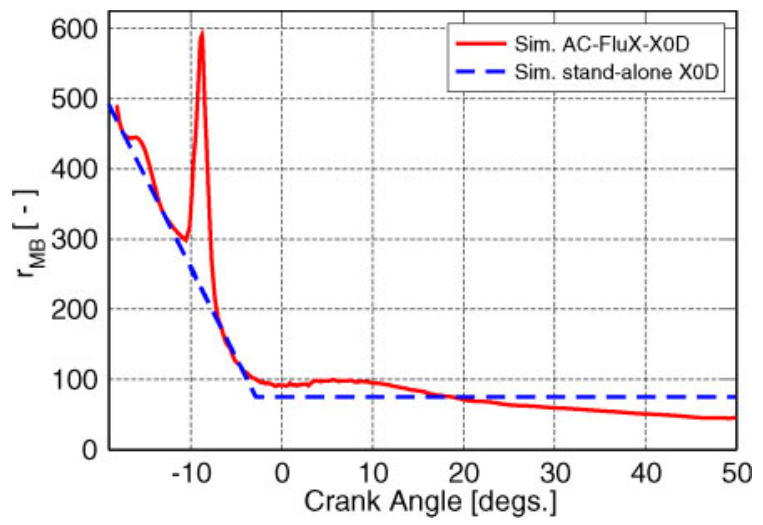

Fig. 13 Average parameter $r_{\mathrm{MB}}$ as evaluated from the interactively coupled CFD-multi-zone approach and as incorporated into the standalone multi-zone model for TS-3 tion of information on fuel injection and vaporization into the stand-alone multi-zone model and the simplified mixing model lead to a fuel mixture fraction distribution that is qualitatively similar to the one from the 3D approach.

The simulation with the interactively coupled CFD-multi-zone approach allows for evaluating the choice of the parameter $r_{\mathrm{MB}}$ in equation (17). The mass exchange rate $\dot{m}_{i k}$ of zone $i$ with neighbouring zone $k$ is computed according to equations (9) and (10). By inserting the mass exchange rate $\dot{m}_{i k}$ into equation (17), a corresponding individual mixing parameter $r_{\mathrm{MB}, i k}$ can be determined which describes the level of mixing between zones $i$ and $k$. Averaging the individual mixing parameters $r_{\mathrm{MB}, i k}$ yields the parameter $r_{\mathrm{MB}}$.

Figure 13 displays the average parameter $r_{\mathrm{MB}}$ around TDC firing for TS-3, as accordingly evaluated from the simulation with the interactively coupled CFD-multi-zone approach and as incorporated into the stand-alone multi-zone model. For incorporation, $r_{\mathrm{MB}}$ was set to 75 during compression and expansion, with a linear increase up to 500 during injection and a linear decrease back again to 75 after end of injection. Qualitatively, both evolutions are similar. Altogether, $r_{\mathrm{MB}}$ decreases after end of injection (EOI) with decaying turbulence intensity after EOI. The evolution of $r_{\mathrm{MB}}$ as evaluated from the interactively coupled CFD-multi-zone approach reveals two peaks. These two peaks are due to first and second stage ignition. The corresponding integral average of the whole simulation is equal to 169.7 .

The SOI variations TS- 1 to TS-5 were also investigated in a previous work by Felsch et al. [48], with one constant value of 100 being chosen for $r_{\mathrm{MB}}$, which is of the same order of magnitude as 169.7. In reference [48], the overall simulation results for the stand-alone multi-zone model in terms of pressure curves, $\mathrm{IMEP}_{\mathrm{HP}}$, CA10, CA50, CO emissions, combustion efficiency, and $\mathrm{NO} / \mathrm{NO}_{x}$ emissions were not as accurate in predicting the experimental results as the ones presented in the current work, where the evolution of $r_{\mathrm{MB}}$ was qualitatively adjusted to the one from the 3D approach.

It should be emphasized, too, that the order of magnitude of the parameter $r_{\mathrm{MB}}$ strongly depends on the level of premixing. In reference [27], where more advanced injection timings were investigated, a constant value of six was sufficient for $r_{\mathrm{MB}}$. Therefore this modelling parameter must be adjusted for each engine type.

Finally, the left-hand side of Fig. 14 compares the pressure trace obtained from the stand-alone multi- 

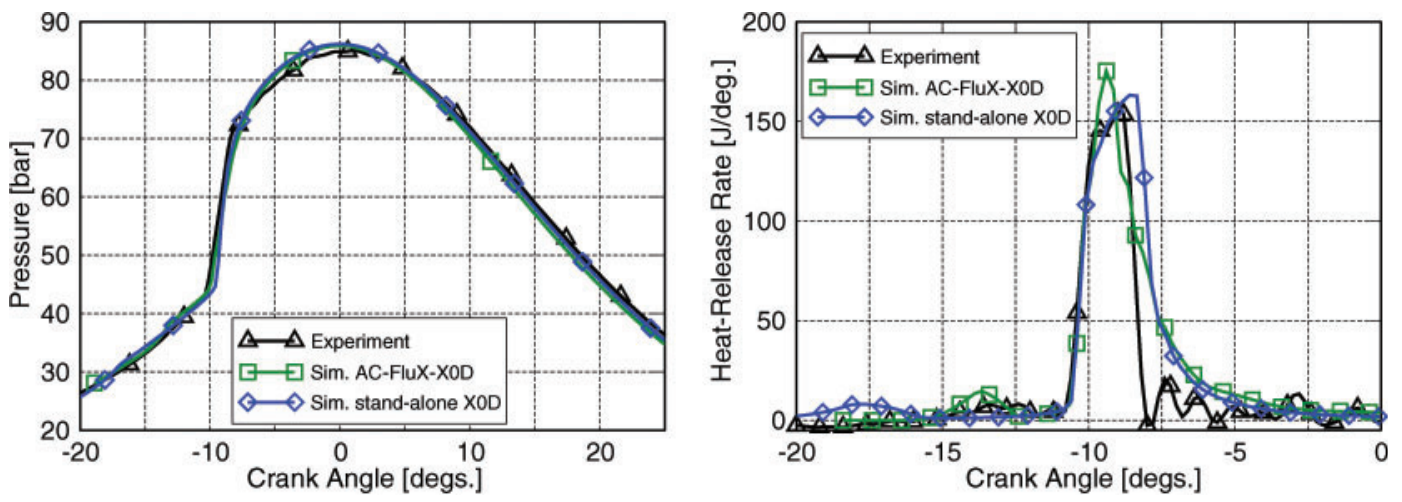

Fig. 14 Average cylinder pressure comparison (left) and average apparent net heat-release rate comparison (right) for stand-alone multi-zone model to the interactively coupled CFDmulti-zone approach and the experiment for TS-3
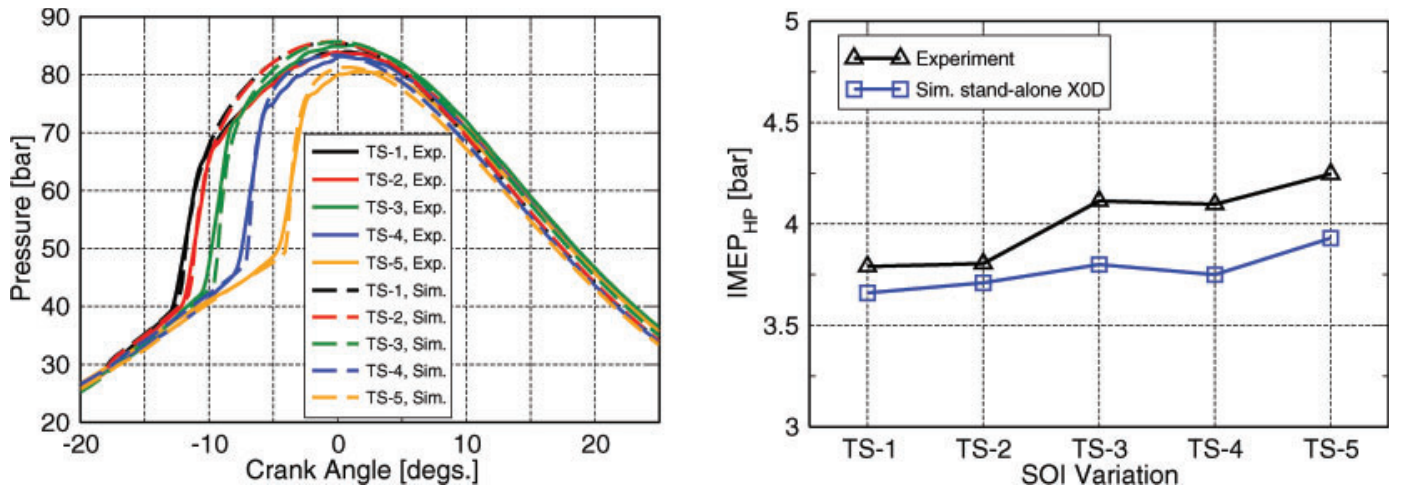

Fig. 15 Average cylinder pressure (left) and $\operatorname{IMEP}_{\mathrm{HP}}$ (right) for the SOI variation. Comparison between simulation with the stand-alone multi-zone model and experiment

zone model with the one obtained from the interactively coupled CFD-multi-zone approach and the experimental data. All three pressure curves match very well. This also holds for the comparison of the corresponding average apparent net heat-release rates shown on the right-hand side of Fig. 14 .

Figures $10,11,12,13$, and 14 reveal that systematically reducing the interactively coupled CFDmulti-zone approach according to the procedure

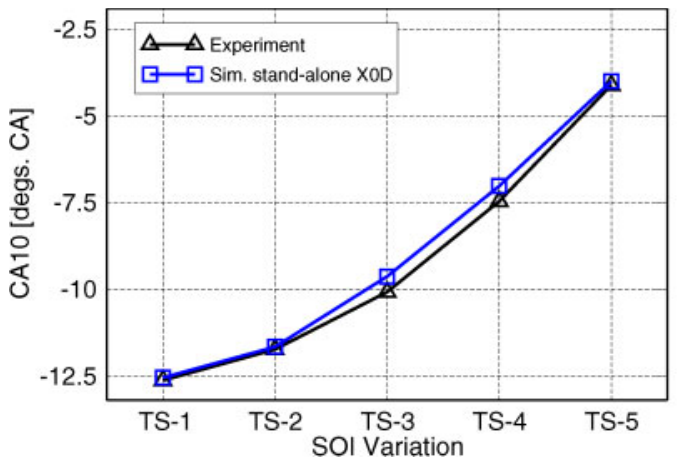

outlined in section 2.4 leads to a reliable stand-alone multi-zone model that can be used to efficiently model PCCI combustion. This is further shown in the following.

\subsubsection{SOI variation}

Similar to section 4.1.2, Fig. 15 first compares the pressure curves and the corresponding IMEP $_{\mathrm{HPS}}$

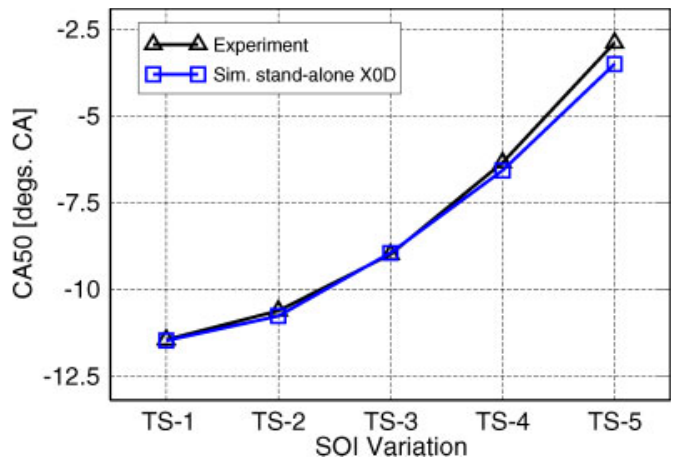

Fig. 16 CA10 (left) and CA50 (right) for the SOI variation. Comparison between simulation with the stand-alone multi-zone model and experiment 

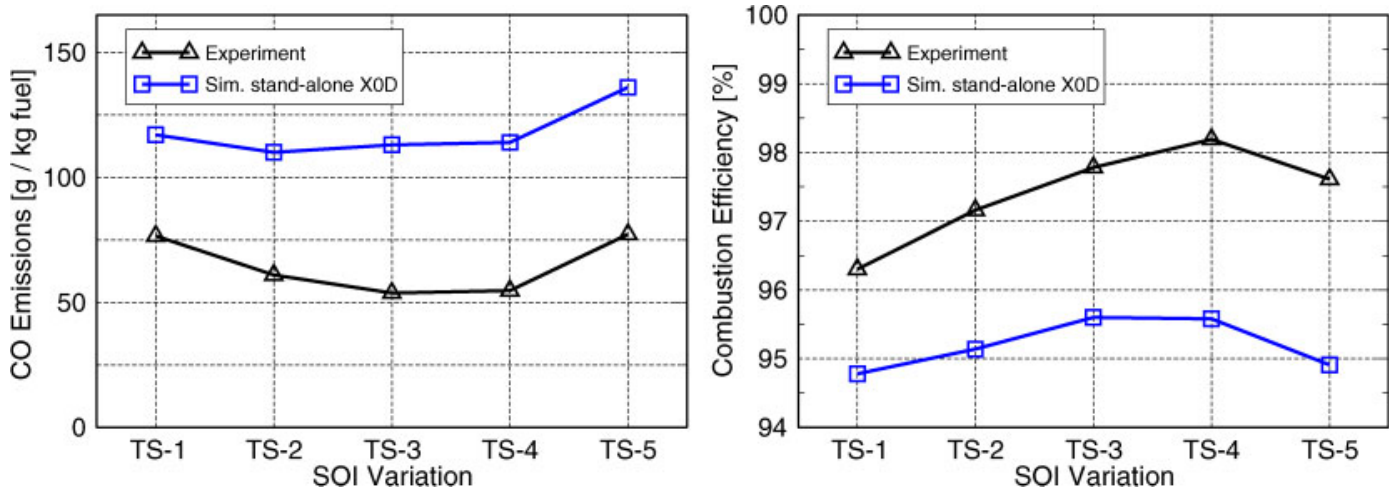

Fig. 17 CO emissions in grammes/kilogramme fuel in the exhaust gas (left) and combustion efficiency in per cent (right) for the SOI variation. Comparison between simulation with the stand-alone multi-zone model and experiment

obtained from the stand-alone multi-zone model simulations with the results from the experiments for the complete SOI variation TS-1 to TS-5. Again, for all cases the experimental pressure curves and the experimental $\mathrm{IMEP}_{\mathrm{HP}} \mathrm{S}$ closely agree with the stand-alone multi-zone model. There is only a slight overestimation of the maximum peak pressure and a slight underestimation of the $\mathrm{IMEP}_{\mathrm{HP}}$ in all simulations.

Figure 16 shows CA10 and CA50 for all experiments and simulations. Once more, CA10 and CA50 are in very good agreement with the experimental results.

Figure 17 summarizes the CO emissions in grammes/kilogramme fuel in the exhaust gas and the combustion efficiency in per cent for the complete SOI variation. The simulations overestimate the $\mathrm{CO}$ emissions and underestimate the combustion efficiency. The qualitative agreement

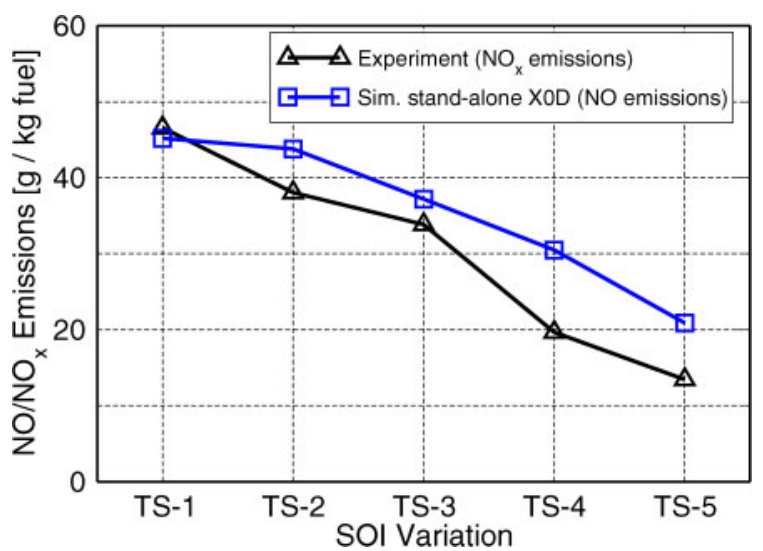

Fig. $18 \mathrm{NO} / \mathrm{NO}_{x}$ emissions in grammes/kilogramme fuel in the exhaust gas for the SOI variation. Comparison between simulation with the stand-alone multi-zone model and experiment for the $\mathrm{CO}$ emissions and the combustion efficiency over the whole SOI variation is good. The HC emissions (not shown here) are captured reasonably.

In Fig. 18, a comparison between numerically and experimentally obtained $\mathrm{NO} / \mathrm{NO}_{x}$ emissions in grammes/kilogramme fuel in the exhaust gas is presented for the complete SOI variation. There is good agreement.

\section{GAS EXCHANGE}

The stationary validation described in the previous section is restricted to the high-pressure part of the engine cycle. The use of the stand-alone multi-zone model within a closed-loop controller for PCCI combustion requires that it is capable of predicting the dependency of the controlled variables on the actuators. As already mentioned, the actuators are the SOI, the external EGR rate, and the total fuel mass injected. The controlled variables are the IMEP and CA50. The latter is directly obtained from a simulation with the stand-alone multi-zone model. The former, however, can only be predicted for the time frame from the closing of the intake valve until opening of the exhaust valve. For this reason, the calculation of the IMEP $_{\mathrm{HP}}$ is extended to the gas exchange part of the engine cycle. This extension is described in the present section.

The IMEP of the whole engine cycle is composed of the high-pressure part and the part describing the losses caused by the gas exchange. From the viewpoint of automatic control, a model predicting the accumulated losses occurring from EVO until IVC is sufficient. Furthermore, as the aim of this work is model reduction, the lowest acceptable approach in terms of detail level and calculation time is desired. Thus, a mean value model for the gas exchange is presented in 
the following. Nevertheless, if desirable, many commercially available software packages exist for a more detailed calculation of the gas exchange part of the engine cycle (e.g. WAVE [49] or GT-POWER [50]).

The calculation of the IMEP throughout the gas exchange (IMEP ${ }_{\mathrm{GE}}$ ) is physically inspired by pumping losses. This approach is derived from the one proposed in reference [51]. It was introduced in Hoffmann et al. [52]. The formulation is valid for a constant engine speed. However, for the test case investigated, this restriction related to the engine speed can be disregarded, because the model validation is carried out at a constant engine speed of $2000 \mathrm{r} / \mathrm{min}$. The $\mathrm{IMEP}_{\mathrm{GE}}$ is given by

$$
\begin{aligned}
\operatorname{IMEP}_{\mathrm{GE}}= & x_{1} \times\left(p_{\mathrm{ae}}-p_{\mathrm{be}}\right)+x_{2} \times \sqrt{p_{\mathrm{ae}}-p_{\mathrm{be}}} \\
& +p_{\mathrm{be}} \times\left(x_{3} \times \frac{p_{\mathrm{be}}}{T_{\mathrm{be}}}+x_{4} \times \sqrt{\frac{p_{\mathrm{be}}}{T_{\mathrm{be}}}}\right) \\
& +x_{5} \times \sqrt{T_{\mathrm{ae}}-T_{\mathrm{be}}}+x_{6}
\end{aligned}
$$

where $p_{\text {ae }}$ is the pressure in the exhaust manifold (after engine), $p_{\mathrm{be}}$ is the pressure in the intake manifold (before engine), $T_{\mathrm{ae}}$ is the temperature in the exhaust manifold, and $T_{\mathrm{be}}$ is the temperature in the intake manifold.

Equation (19) includes terms of the volume flow through an orifice and through a throttle, as well as the dependency on the volumetric efficiency or on the density at the intake, respectively. The latter is represented by the fraction $p_{\mathrm{be}} / T_{\mathrm{be}}$, which is related to the ideal gas law $\rho=p / R T=$ const. $\times p / T$. The engine-dependent constants $x_{1}, x_{2}, x_{3}, x_{4}, x_{5}$, and $x_{6}$ need to be determined by a fitting against experimental data.

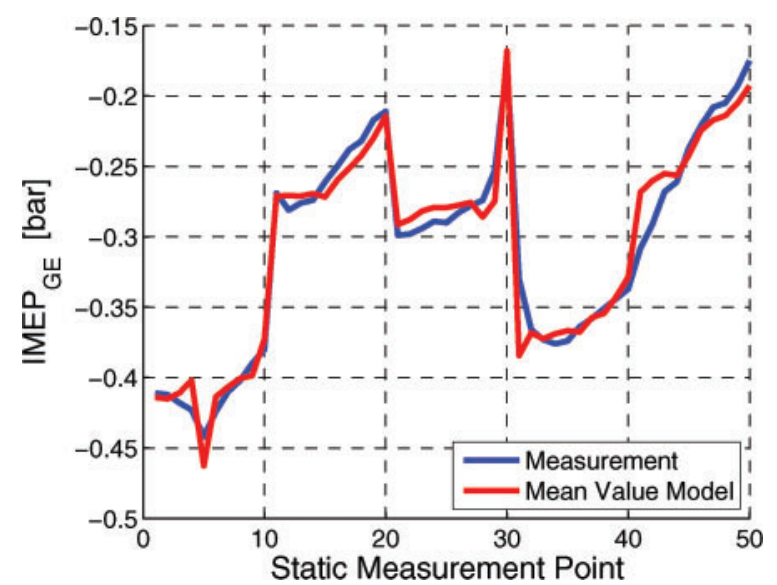

Fig. 19 IMEP $_{\mathrm{GE}}$ of the gas exchange for all 50 operating conditions presented in section 3.1.2. Comparison between mean value model calculation and experiment

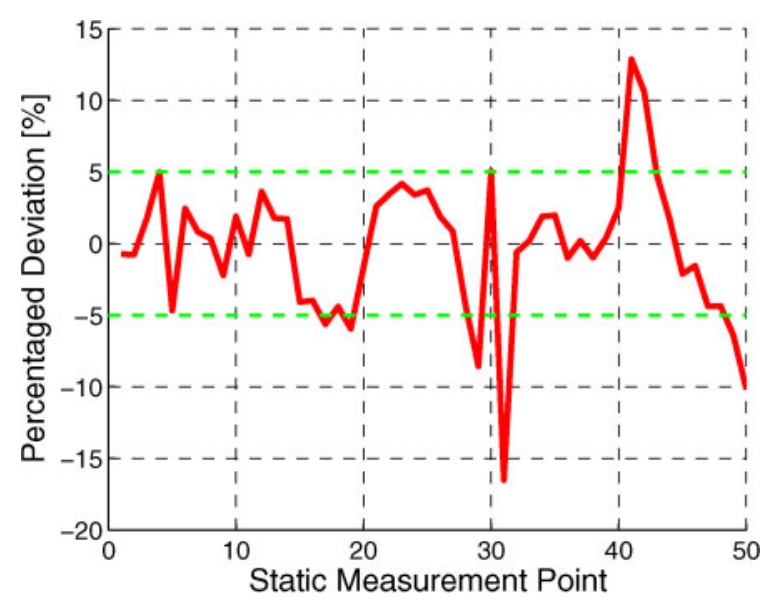

Fig. 20 Percentaged deviation of the calculated $\mathrm{IMEP}_{\mathrm{GE}}$ of the gas exchange from all 50 operating conditions presented in section 3.1.2

The mean value model for the gas exchange depends on the state directly after and before the combustion process or the manifolds after and before the engine, respectively. In equation (19), these states are denoted with the subscripts 'ae' and 'be'. Inputs to the model from the exhaust manifold (i.e. $p_{\mathrm{ae}}$ and $T_{\mathrm{ae}}$ ) can be taken from the high-pressure calculation with the stand-alone multi-zone model, while the inputs to the model from the intake manifold (i.e. $p_{\text {be }}$ and $T_{\text {be }}$ ) become influencing variables of the plant model. These influencing variables can serve as a disturbance input within the closed-loop control.

After appropriately fitting the constants $x_{1}$ to $x_{6}$, the approach is capable of predicting the $\mathrm{IMEP}_{\mathrm{GE}}$ within a 5 per cent accuracy for most of the 50 operating conditions presented in section 3.1.2. This is shown in Figs 19 and 20. Combining the standalone multi-zone model X0D with this gas exchange model therefore leads to a static model, which can be used to determine the static dependency of the controlled variables IMEP and CA50 on the actuated variables SOI, external EGR rate, and total fuel mass injected. Influencing inputs are the pressure and temperature in the intake manifold.

\section{SYSTEM IDENTIFICATION}

\subsection{Wiener model}

The combination of the stand-alone multi-zone model and mean value model for the $\mathrm{IMEP}_{\mathrm{GE}}$ of the gas exchange does so far not include any dynamics of the controlled variables. Hence, a structure has to be chosen which is suitable for 


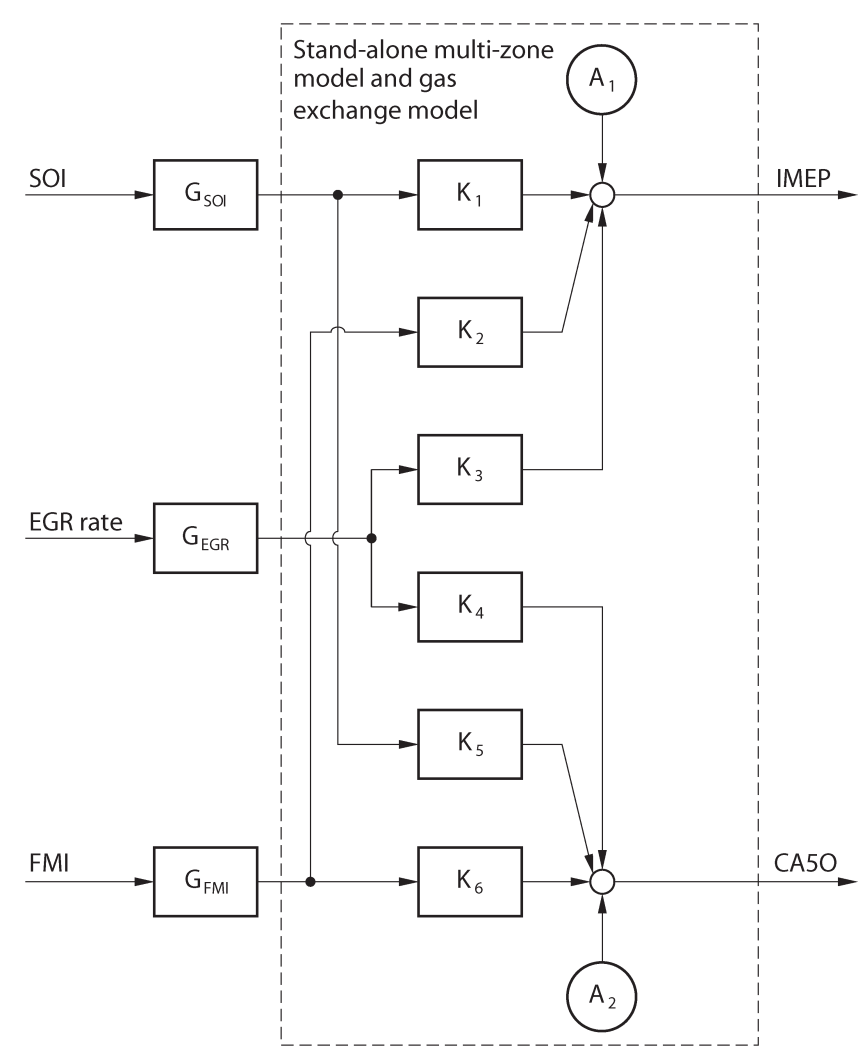

Fig. 21 Model structure used for identifying the system's dynamic time-discrete transfer functions. The dashed line shows the part that may be substituted by the complete integrated static model (see section 7.1)

adding the dynamic aspect to the static model. In control theory, two types of models or a combination of both have been established for this purpose: Wiener and Hammerstein models $[\mathbf{5 3}, \mathbf{5 4}]$. Both have in common a separation of a dynamic transfer function from the system's non-linear static transfer behaviour. With Hammerstein models, the system's static non-linearity is modelled before, with Wiener models after the system's dynamics. As the dynamic physical behaviour of the real engine shall be enforced on the whole static model, a Wiener-type dynamics is desired [52]. With this choice, the inputs to the static model are overlaid with time attributes, which enforces the dynamic behaviour on the combustion simulation. Thus, IMEP and CA50 are consequently affected by the dynamics to be identified.

Figure 21 depicts the implemented Wiener-type dynamics. Here, the static non-linear behaviour is described by the combined integrated static model, or alternatively the gains $K_{1}$ to $K_{6}$ and the two offset values $A_{1}$ and $A_{2}$, respectively. For the system identification described in this section, the latter alternative is used. Therefore, the gains $K_{1}$ to $K_{6}$ and the two offset values $A_{1}$ and $A_{2}$ are dependent on the operating point due to the system's non-linearity.

As only load-transient conditions with a static engine speed are regarded as a first step, the identified dynamics is assumed to be given by a $P T_{1}$. If speedtransient conditions would additionally apply, this approach would either have to be extended by time constants tabulated over engine speed or by a physical model for the mechanical dynamics of pistons, conrods, crank, and additionally gas exchange, respectively. The total model would still have to be fitted to measurements as well. With the latter approach, the unknown masses and inertias of the drivetrain, as well as parameters of the gas dynamics need to be identified. An example for such an approach is the work by Schulze et al. [55].

\subsection{Step response experiments}

A description of the dynamics from the system's inputs SOI, external EGR rate, and total fuel mass injected (FMI) towards the outputs IMEP and CA50 is needed. For this purpose, several approaches for arranging experiments exist in the literature. In this regard, step responses are the most common way for the identification of a system's dynamics. The experimental setup (see also section 3.1) realizes this by using an ES1000 system by ETAS as the central unit for impressing the steps on the actuated variables, as well as for simultaneously collecting the data of the step responses in the controlled variables. More details regarding this Rapid Control Prototyping system can be found in reference [56]. The steps in the actors were enabled by an Emulator-Tastkopf (ETK)-connection of the ES1000 system to the engine's type EDC16 electronic control unit (ECU). The physical actor for the SOI and the total FMI obviously is the common-rail direct injector, while for the EGR rate there is no explicit acting device. For this, the EGR rate was changed by the combination of the actors EGR valve and variable geometry turbine (VGT) position. It is impossible to make the engine step in EGR rate. Nevertheless, the system shows a dynamic response to a change in the EGR rate caused by a simultaneous step in EGR valve and VGT position. For the operating points to step between, the corresponding values for EGR throttle and VGT position were determined for a steady-state operation. Furthermore, the test bed offers no direct possibility to measure the dynamics of the EGR rate, which is the input and actuating variable for the static model. Commonly, in modern ECUs in series applications, the mass flow of fresh air aspirated by 
the engine is measured instead [57]. The EGR rate can be calculated by

$$
\text { EGR rate }=\frac{\dot{m}_{\mathrm{EGR}}}{\dot{m}_{\text {trapp. mass }}}=\frac{\dot{m}_{\text {trapp. mass }}-\dot{m}_{\text {air }}}{\dot{m}_{\text {trapp. mass }}}
$$

where $\dot{m}_{\text {air }}$ denotes the mass flow of fresh air. The total trapped gas mass $\dot{m}_{\text {trapp. mass }}$ mainly depends on the engine speed, as well as on the pressure and temperature in the intake manifold. The engine was run at a constant speed. In addition, the intake manifold conditions did not vary significantly during the step response experiments. The total trapped gas mass $\dot{m}_{\text {trapp. mass }}$ is therefore considered approximately constant and, for this reason, the main dynamics affecting the EGR rate in this case is the same but negative dynamics as with the fresh air mass $\dot{m}_{\text {air. }}$ This was measured by the engine's ECU, but also handed over to the measuring device ES1000 via ETK. Because the steady-state value for the EGR rate is known before and after applying the step in VGT position and EGR valve, the dynamic EGR rate during the tests can be recalculated and used as an input to the model.

Another prerequisite for the system identification is the measurement of the target values or outputs of the model, respectively. Both IMEP and CA50 are calculated from the pressure progression measurement. This has to be realized by an additional device, as in series applications no pressure analysis is implemented in the ECU. In this work, a FI2RE system by IAV was used [58]. It analyses the pressure signal in real time and hands it over to the measurement system ES1000 via a controller area network (CAN) bus.

\subsection{Dynamic transfer functions}

The step response experiments provide a dataset containing the temporally resolved signals for SOI, external EGR rate, and FMI, as well as the corresponding response in IMEP and CA50. The aim of the extraction of the system's dynamics is the previously mentioned Wiener-type dynamics. The advantage of this approach is also its disadvantage. By assigning the system's dynamics to the inputs, a relatively simple model structure concerning the dynamic transfer functions is achieved, as their number is reduced to the number of inputs (see Fig. 21). However, this also is the main drawback of the approach, because all three inputs influence both outputs. This results in six dynamic dependencies, which have to be described by three transfer functions. The dynamic effects on IMEP and CA50 excited by the changes in SOI thus have to be represented by a transfer function $G_{\text {SOI }}$, the dynamic influence on both outputs caused by changes in the external EGR rate or the total FMI by a transfer function $G_{\mathrm{EGR}}$ or $G_{\mathrm{FMI}}$, respectively.

The transfer function $G_{\text {SOI }}$ can be determined from the evaluation of the responses of IMEP and CA50 to steps in SOI. The same sampling rate as used during the measurements with the ES1000 system was used for the time-discrete transfer function. The sampling rate $T_{\mathrm{s}}$ of the calculation of IMEP and CA50 is preset by the engine's speed and is given by

$$
T_{\mathrm{s}}=\frac{2 \times 60 \mathrm{~s} / \mathrm{min}}{n_{\text {eng. } .}[\mathrm{r} / \mathrm{min}]}
$$

With $n_{\text {eng. }}=2000 \mathrm{r} / \mathrm{min}$, a new value for IMEP and CA50 is calculated every $0.06 \mathrm{~s}$. To ensure compliance with Shannon's theorem, the measurements were sampled with $0.01 \mathrm{~s}$, and consequently the same sample time was chosen for the time-discrete transfer function.

In mathematics and signal processing, the $Z$ transform converts a discrete time-domain signal, which is a sequence of real or complex numbers, into a complex frequency-domain representation [59]. It is the discrete equivalent of the Laplace transform. The discrete time-domain function $f\left(k \times T_{\mathrm{spl}}\right)$ of sample time $T_{\mathrm{spl}}$ is transformed to a $z$-domain transfer function $F(z)_{\left(T_{\mathrm{spl}}\right)}$ valid for the specified sample time. In this representation, $z$ can be understood as a time shift operator: $x\left(k \times T_{\mathrm{spl}}-n \times T_{\mathrm{spl}}\right)$ $\sim-z^{-n} X(z)_{\left(T_{\mathrm{spl}}\right)}$. The measurements indicate the use of a $P T_{1}$-transfer function. With a sample time of $0.01 \mathrm{~s}$ the identified $P T_{1}$ is given by

$$
G(z)_{\mathrm{SOI},(0.01)}=\frac{\widehat{\mathrm{SOI}}}{\mathrm{SOI}}=\frac{0.1}{z-0.9}
$$

Here, the superscript ' $\cdots$ ' indicates the temporal attribute of the signal. Figure 22 shows the results obtained with this transfer function. Shown is the response of IMEP and CA50 in measurement and system identification to various steps in SOI. For the system identification, the corresponding static gains $K_{1}$ to $K_{6}$ and the two offset values $A_{1}$ and $A_{2}$ were adjusted to the operating point to substitute the complete integrated static model. The results indicate that the time constant of the chosen transfer function is suitable to describe the engine's temporal attribute regarding a change in SOI.

The sampling rate used here is obviously not consistent with the engine speed. The static integrated 

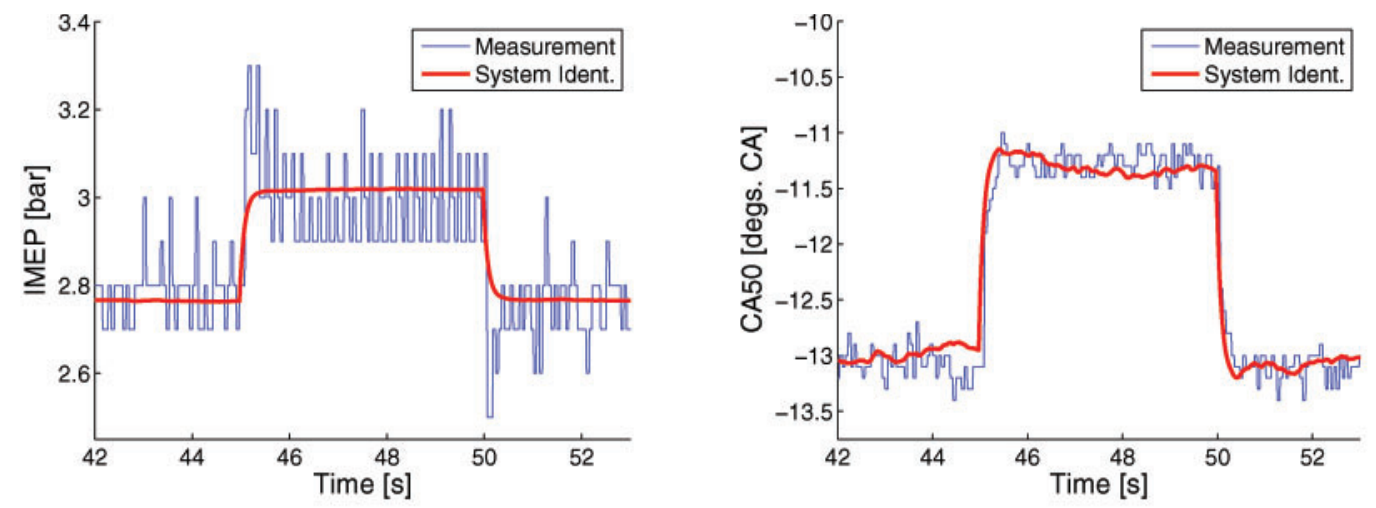

(a) IMEP (left) and CA50 (right) for an SOI step from $-40.7^{\circ}$ to $-30.7^{\circ} \mathrm{CA}$ aTDC and back with an external EGR rate of 30 per cent and an injected fuel mass of $10.2 \mathrm{mg} / \mathrm{cycle}$
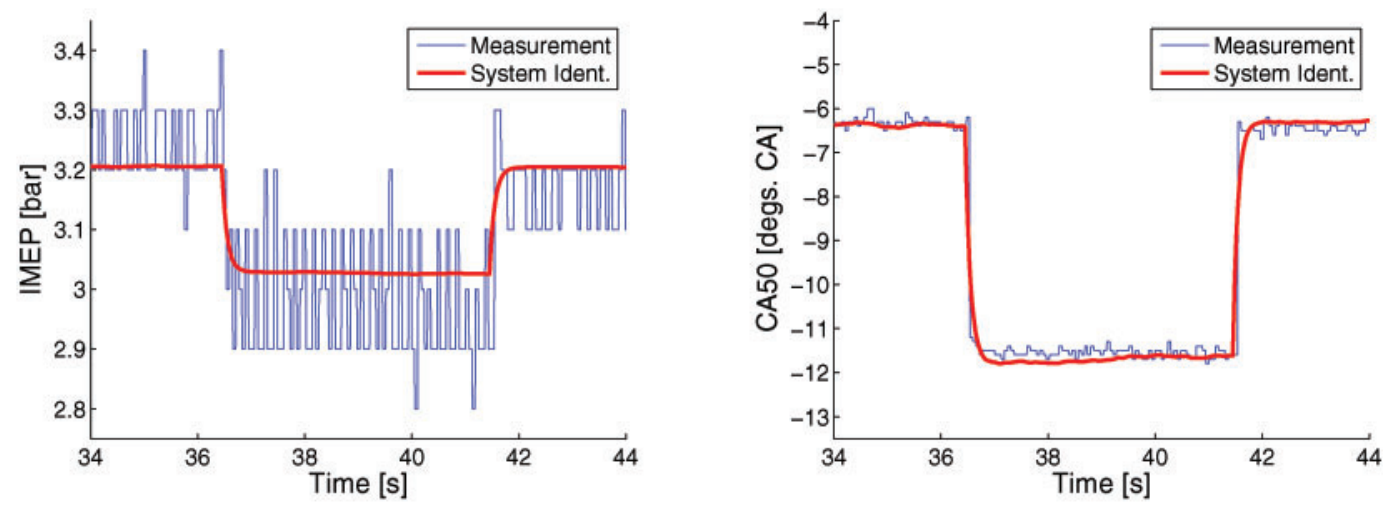

(b) IMEP (left) and CA50 (right) for an SOI step from $-20.7^{\circ}$ to $-30.7^{\circ} \mathrm{CA}$ aTDC and back with an external EGR rate of 30 per cent and an injected fuel mass of $10.2 \mathrm{mg} /$ cycle
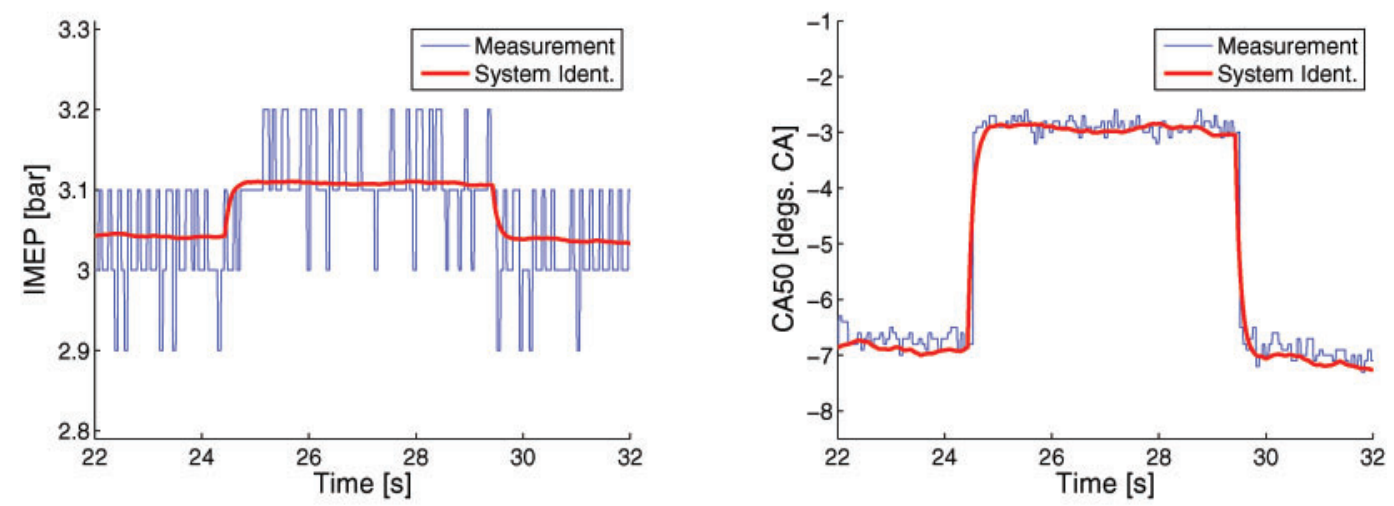

(c) IMEP (left) and CA50 (right) for an SOI step from $-30.7^{\circ}$ to $-20.7^{\circ} \mathrm{CA}$ aTDC and back with an external EGR rate of 45 per cent and an injected fuel mass of $10.2 \mathrm{mg} / \mathrm{cycle}$

Fig. 22 Comparison between experiment and system identification with identified discrete transfer function $G(z)_{\text {SOI, }}(0.01)$

model is executed every engine cycle, demanding a sample time of $0.06 \mathrm{~s}$ for $2000 \mathrm{r} / \mathrm{min}$. To remedy this problem and to enable the implementation of the transfer function into the integrated model, the timediscrete transfer function is resampled with the new sample time of $0.06 \mathrm{~s}$ to

$$
G(z)_{\text {SOI, }(0.06)}=\frac{\widehat{\text { SOI }}}{\text { SOI }}=\frac{0.4686}{z-0.5314}
$$


In an analogous manner, the transfer functions $G(z)_{\mathrm{EGR}}$ and $G(z)_{\mathrm{FMI}}$ can be determined from the evaluation of the responses of IMEP and CA50 to the dynamic EGR rate or dynamic total FMI signal, respectively. Therefore, the EGR rate signal is recalculated as described above. The corresponding gains and offset values in Fig. 21 are again adjusted such that the static non-linear transfer behaviour is met. The fitting of the system's dynamics is evaluated with a measuring sample time of $0.01 \mathrm{~s}$ and resampled to $0.06 \mathrm{~s}$ afterwards.

The influence of the external EGR rate is given by

$$
G(z)_{\mathrm{EGR},(0.01)}=\frac{\mathrm{EGR} \text { rate }}{\text { EGR rate }}=\frac{0.015}{z-0.985}
$$

and can be resampled with $T_{\mathrm{s}}=0.06 \mathrm{~s}$ to

$$
G(z)_{\mathrm{EGR},(0.06)}=\frac{\mathrm{EGR} \text { rate }}{\mathrm{EGR} \text { rate }}=\frac{0.08669}{z-0.9133}
$$

Using $G(z)_{\mathrm{EGR}}(0.01)$, the results shown in Fig. 23 were achieved.

For the dynamic dependency of the model's outputs on the total FMI the transfer function

$$
G(z)_{\mathrm{FMI},(0.01)}=\frac{\widehat{\mathrm{FMI}}}{\mathrm{FMI}}=\frac{0.071}{z-0.9290}
$$

was determined, which leads to the resampled transfer function

$$
G(z)_{\mathrm{FMI},(0.06)}=\frac{\widehat{\mathrm{FMI}}}{\mathrm{FMI}}=\frac{0.3572}{z-0.6428}
$$

Figure 24 demonstrates the achieved result for various steps in FMI modelled by equation (26).

Note that in this case there are two active influences, as it is impossible to only change the value for FMI and keep the EGR rate constant without adapting the settings of EGR valve and VGT position. Therefore, the dependencies of IMEP and CA50 on FMI and on the EGR rate have to be fitted simultaneously.

\section{INTEGRATED MODEL}

In the previous three sections, all discussed model parts (stand-alone multi-zone model, gas exchange model, and dynamic time response) were each validated separately. In this section, the realization of an integrated model composed of these three parts within a suitable test environment for control applications is described. Afterwards, the integrated model is validated against transient experimental data. This section closes with a discussion of this model's potential in the framework of closed-loop control development.

\subsection{Suitable test environment for control applications}

For application within a closed-loop control simulation, the stand-alone multi-zone model was transferred to an environment suitable for the conception and testing of controllers. For this study, the MATLAB/Simulink environment of The MathWorks, Inc. was chosen as an appropriate platform [60]. The stand-alone multi-zone model X0D written in FORTRAN 77 was embedded into a MATLAB/Simulink FORTRAN s-function enabling the simulation of the stand-alone multi-zone model from within MATLAB/ Simulink. Moreover, the gas exchange model was implemented into this FORTRAN s-function. The three dynamic transfer functions were added by means of time-discrete $P T_{1}$-dynamics within appropriate function blocks. The MATLAB/Simulink setup is visualized in Fig. 25.

The simulation environment is realized such that the initial in-cylinder conditions reflect the engineout conditions of the prior cycle. This establishes the cycle-to-cycle connectivity. However, as the external EGR was cooled (see also section 3.1.2), the measurements did not show a strong dependency of the intake conditions on the engine-out conditions, except for the external EGR composition when varying the total fuel mass injected. Aside from the initial in-cylinder conditions, the numerical setup for the multi-zone model (e.g. start and end of simulation, number of zones, incorporated information, etc.) within the MATLAB/Simulink environment was kept unchanged in comparison to the stand-alone multi-zone model simulation setup described in section 3.2.2.

The computational time for the integrated model amounts to approximately $6 \mathrm{~min}$ per engine cycle, using a Dell Latitude D630 notebook with an Intel Core 2 Duo T7500 CPU (dual core with $2.2 \mathrm{GHz}$ ). Obviously, inserting the stand-alone multi-zone model into the MATLAB/Simulink environment does not increase the overall computational time.

\subsection{Transient simulations}

Figure 26 shows the IMEP (left) and the CA50 (right) obtained from the step response experiment, the 

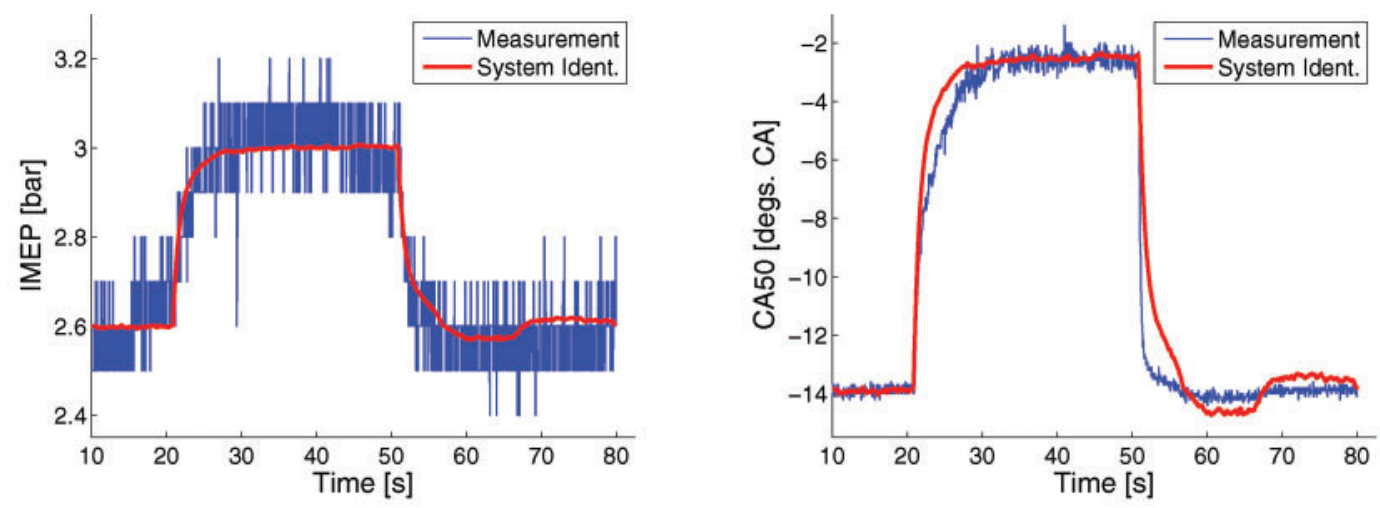

(a) IMEP (left) and CA50 (right) for an EGR step from 30 to 45 per cent and back with an SOI of $-40.7^{\circ} \mathrm{CA}$ aTDC and an injected fuel mass of $10.2 \mathrm{mg} /$ cycle
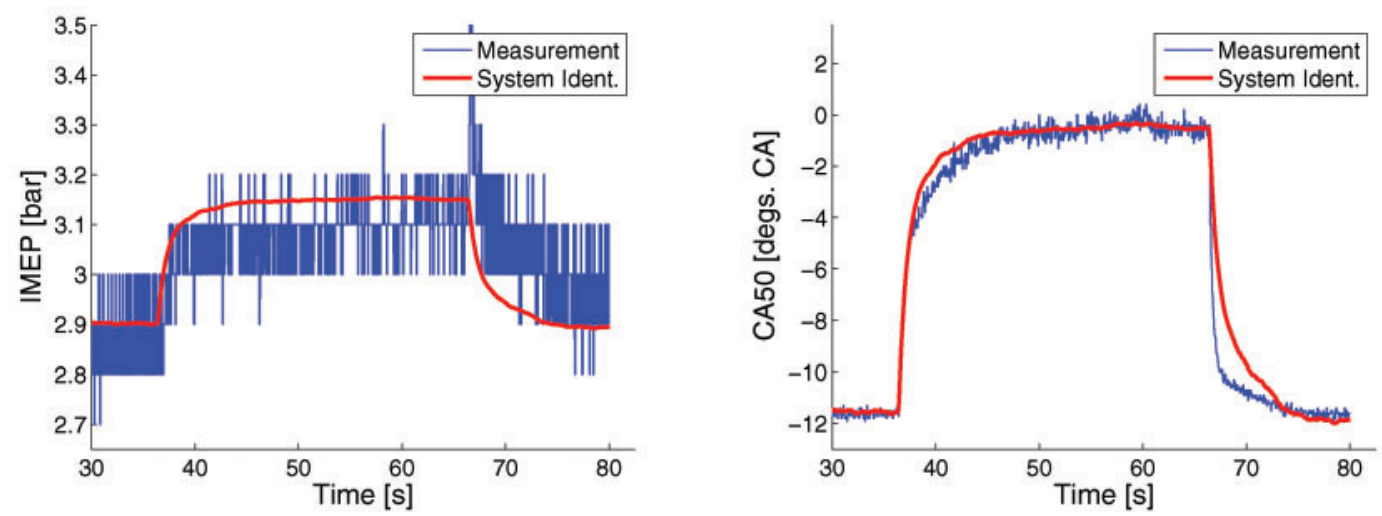

(b) IMEP (left) and CA50 (right) for an EGR step from 30 to 45 per cent and back with an SOI of $-30.7^{\circ} \mathrm{CA}$ aTDC and an injected fuel mass of $10.2 \mathrm{mg} /$ cycle
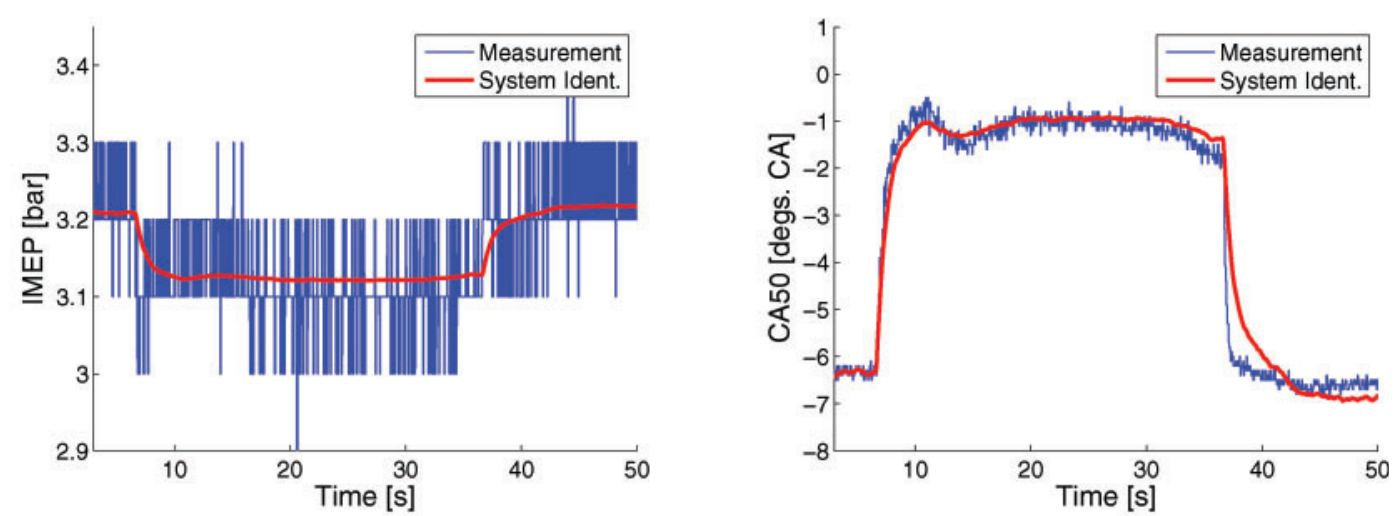

(c) IMEP (left) and CA50 (right) for an EGR step from 30 to 45 per cent and back with an SOI of $-20.7^{\circ} \mathrm{CA}$ aTDC and an injected fuel mass of $10.2 \mathrm{mg} /$ cycle

Fig. 23 Comparison between experiment and system identification with identified discrete transfer function $G(z)_{\mathrm{EGR},(0.01)}$

system identification with the identified discrete transfer function $G(z)_{\text {SOI }(0.01)}$, and the transient simulation with the integrated model using
$G(z)_{\text {SOI, (0.06) }}$ for an SOI step from $-20.7^{\circ}$ to $-30.7^{\circ}$

CA aTDC and back with an external EGR rate of 30 per cent and an injected fuel mass of $10.2 \mathrm{mg} /$ cycle. 

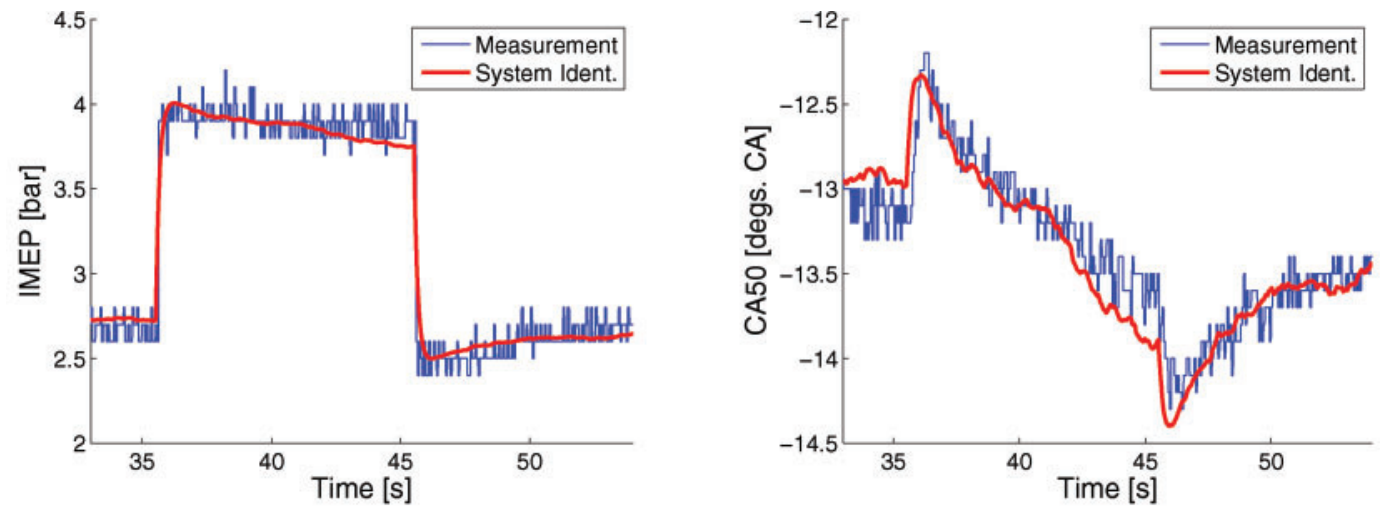

(a) IMEP (left) and CA50 (right) for an injected fuel mass step from 10.2 to $13.6 \mathrm{mg} /$ cycle and back with an $\mathrm{SOI}$ of $-35.7^{\circ} \mathrm{CA}$ aTDC and an external EGR rate of 45 per cent
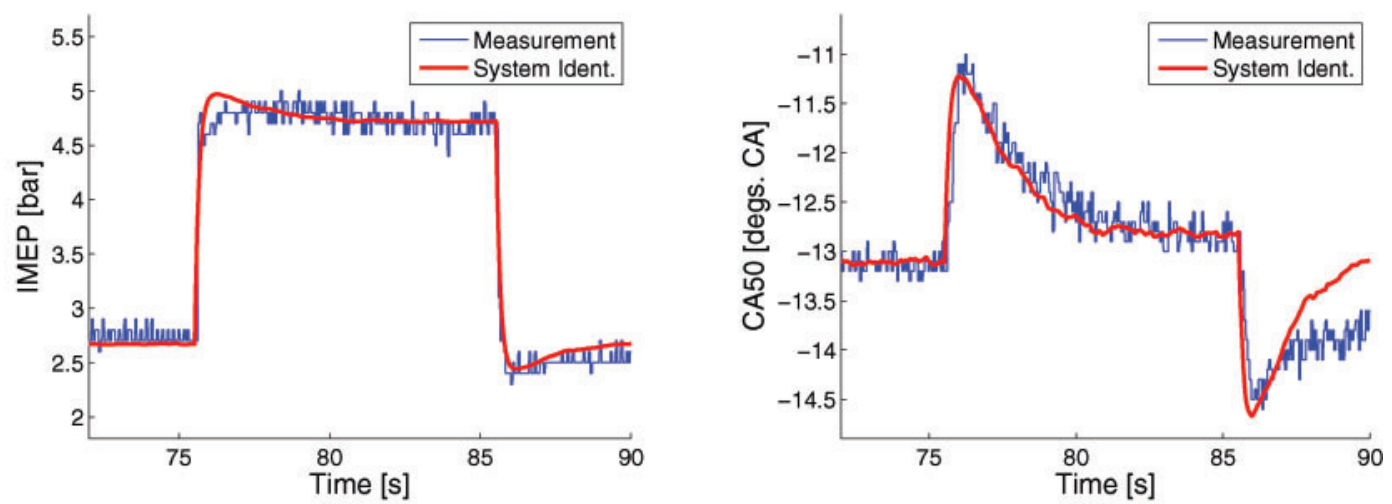

(b) IMEP (left) and CA50 (right) for an injected fuel mass step from 10.2 to $17.0 \mathrm{mg} /$ cycle and back with an

SOI of $-35.7^{\circ} \mathrm{CA}$ aTDC and an external EGR rate of 45 per cent

Fig. 24 Comparison between experiment and system identification with identified discrete transfer function $G(z)_{\mathrm{FMI},(0.01)}$

The results obtained from the step response experiment and the system identification are already shown in Fig. 22(b). The simulation results are in very good agreement with the measurements. In particular, the dynamic step responses are well reproduced. Similar simulation results (not shown

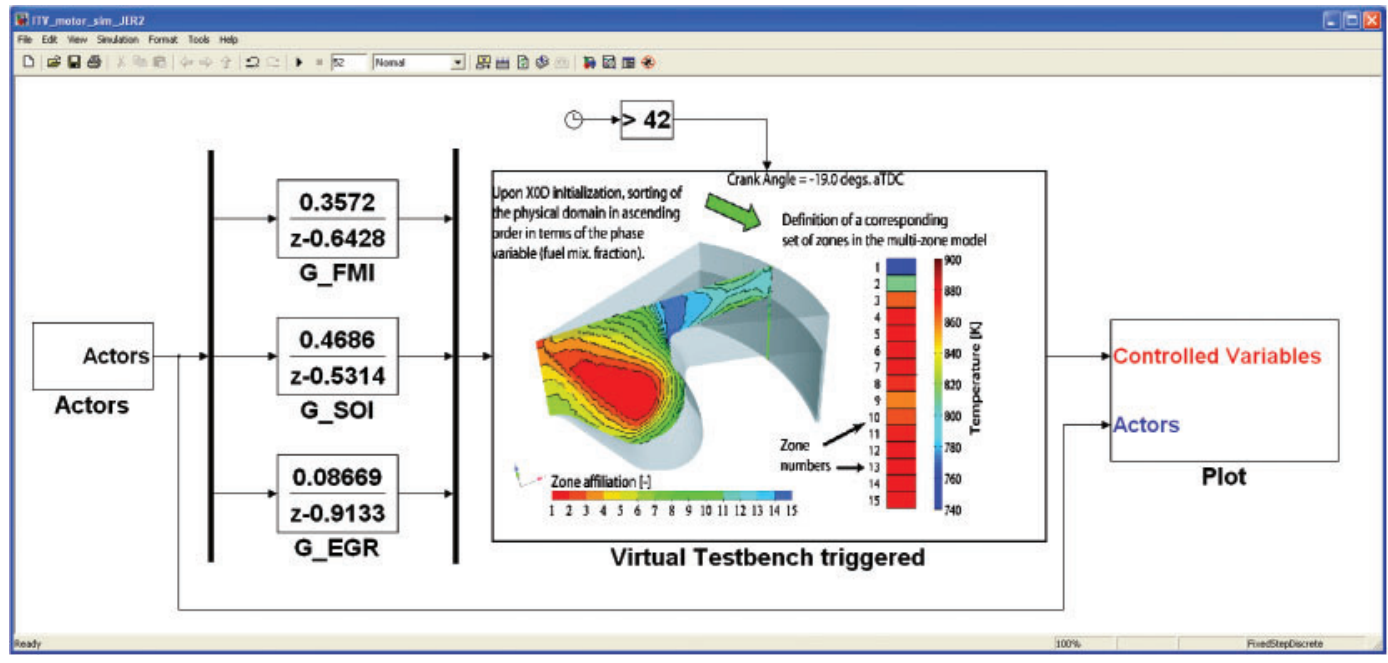

Fig. 25 MATLAB/Simulink setup for transient simulations 

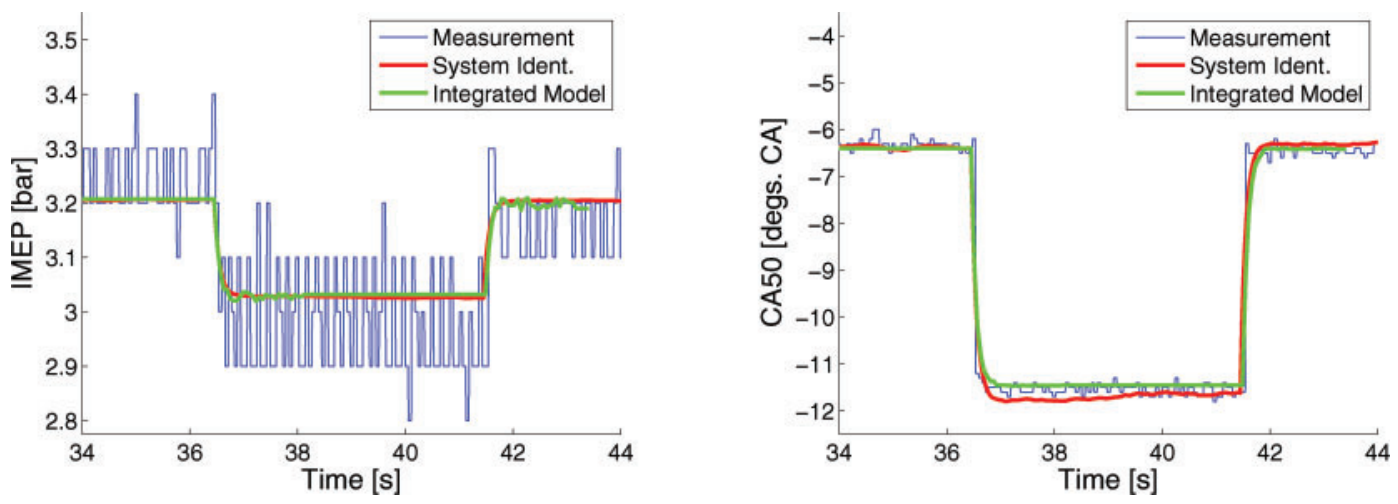

Fig. 26 IMEP (left) and CA50 (right) for an SOI step from $-20.7^{\circ}$ to $-30.7^{\circ}$ CA aTDC and back with an external EGR rate of 30 per cent and an injected fuel mass of $10.2 \mathrm{mg} / \mathrm{cycle}$. Comparison between experiment, system identification with identified discrete transfer function $G(z)_{\text {SOI, (0.01) }}$, and integrated model

here for brevity) were achieved for all other step response experiments presented in section 6.3. This validates the integrated model composed of the stand-alone multi-zone model, the gas exchange model, and the identified system dynamics.

\subsection{Potential within closed-loop control development}

The strict derivation of the stand-alone multi-zone model from a detailed 3D CFD approach integrates the detailed knowledge from combustion simulation tools into closed-loop control. This novel procedure establishes a broad field of possibilities for testing completely new controlled process variables. In this context, Fig. 27 displays further outcomes of the transient simulation for an SOI step from $-20.7^{\circ}$ to $-30.7^{\circ} \mathrm{CA}$ aTDC and back with an external EGR rate of 30 per cent and an injected fuel mass of 10.2 $\mathrm{mg} /$ cycle. The NO emissions, the maximum pressure, the burning time from CA10 to CA90, and the ringing intensity are shown.

The combustion noise level is quantified applying the ringing intensity correlation developed by Eng [61]. This correlation relates the ringing intensity to the maximum pressure rise rate, the maximum pressure, and the speed of sound. The ringing intensity is expressed as

$$
\text { Ringing intensity } \approx \frac{1}{2 \gamma} \frac{\left[\beta\left(\mathrm{d} p / \mathrm{d} t_{\max }\right)\right]^{2}}{p_{\max }} \sqrt{\gamma R T_{\max }}
$$

In equation (28), $\mathrm{d} p / \mathrm{d} t_{\max }$ denotes the maximum pressure rise rate, $p_{\max }$ the maximum pressure, and $T_{\max }$ the maximum temperature. $\beta$ is a scaling factor determined from the experimental data. In this work, it was set to $0.005 \mathrm{~ms}$. In [61], a value of $0.05 \mathrm{~ms}$ was applied.

Figure 27 reveals that the NO emissions increase with advanced SOI timings due to an increasing maximum pressure or maximum temperature, respectively. Advancing the SOI also increases the burning time. This reduces the pressure rise rate, leading to a decreasing ringing intensity. These results indicate the potential of the integrated model in the framework of closed-loop control development. Beyond the controlled variables IMEP and CA50, the integrated model is capable of predicting various additional process variables, with four selected ones being briefly discussed in this subsection. These additional process variables allow for defining further process constraints or may even replace the original controlled variables, depending on the specific control task.

\section{SUMMARY AND CONCLUSIONS}

This paper proposes a new approach for the integration of detailed physical combustion knowledge into closed-loop simulations. Closed-loop simulations are a necessary and common tool in the development process of controllers. A detailed interactively coupled CFD-multi-zone approach was reduced to a stand-alone multi-zone model such that the resulting calculation time could be significantly lowered with only a small penalty in accuracy. The stand-alone multi-zone model is capable of describing the combustion characteristics for the high-pressure part of the engine cycle. The controller to be developed shall actuate SOI, external EGR rate, and total FMI to control the IMEP of the whole 

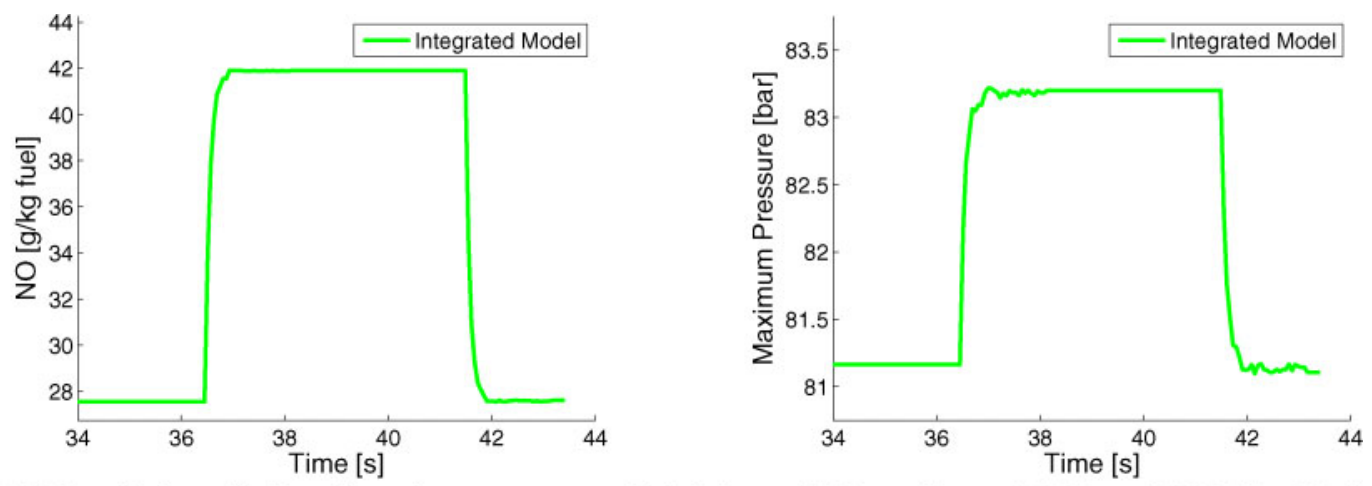

(a) $\mathrm{NO}$ emissions (left) and maximum pressure (right) for an SOI step from $-20.7^{\circ}$ to $-30.7^{\circ} \mathrm{CA}$ aTDC and back with an external EGR rate of 30 per cent and an injected fuel mass of $10.2 \mathrm{mg} / \mathrm{cycle}$
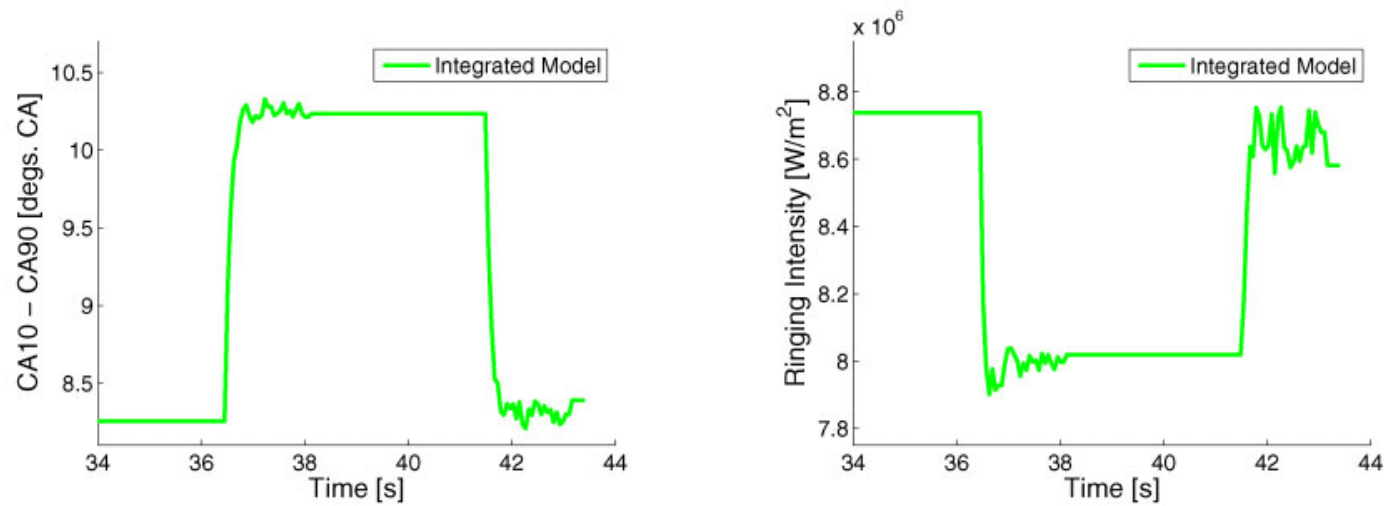

(b) Burning time from CA10 to CA90 (left) and ringing intensity (right) for an SOI step from $-20.7^{\circ}$ to $-30.7^{\circ}$ CA aTDC and back with an external EGR rate of 30 per cent and an injected fuel mass of $10.2 \mathrm{mg} / \mathrm{cycle}$

Fig. 27 Additional process variables obtained from a transient simulation with the integrated model

engine cycle and the CA50. The IMEP ${ }_{\mathrm{HP}}$ of the highpressure engine cycle and the CA50 can be extracted from simulations with the stand-alone multi-zone model. The former was combined with a newly proposed mean value model for the losses of the gas exchange to calculate the IMEP of the whole engine cycle. The combination of these models leads to an accurate static model, which was further extended to a Wiener model for capturing temporal cycle-tocycle dependencies. For every input of the model, a transfer function was determined, forcing the whole model to follow the engine's dynamics. All model parts (stand-alone multi-zone model, gas exchange model, and dynamic time response) were each validated separately. Afterwards, the integrated model composed of all three model parts was validated against transient experimental data.

In the future, the dynamic model will build the framework in which a controller for the PCCI combustion process will be developed. With the developed plant model, this controller will be laid out and tested.

The actuated variable external EGR rate is theoretical as long as there is no corresponding actor to it.
Because of this, the overall goal of this research project (carried out within the Collaborative Research Centre 'SFB 686 - Modellbasierte Regelung der homogenisierten Niedertemperatur-Verbrennung' at RWTH Aachen University, Germany, and Bielefeld University, Germany [62]) is to develop, not only a controller for the PCCI combustion process, but also an additional controller enabling the fastest possible changes in external EGR rate by simultaneously adjusting EGR valve and VGT position [63]. This controller, in combination with its actuated variables EGR valve and VGT position, will serve as the virtual actor for the external EGR rate.

\section{ACKNOWLEDGEMENTS}

The authors would like to thank Bruno Kerschgens from RWTH Aachen University for supporting the numerical simulations and Abhinav Sharma from RWTH Aachen University for supporting the evaluation of the experimental data.

This work was funded by the German Research Foundation (DFG) within the Collaborative Research 
Centre 'SFB 686 - Modellbasierte Regelung der homogenisierten Niedertemperatur-Verbrennung' at RWTH Aachen University, Germany, and Bielefeld University, Germany [62].

\section{(c) Authors 2009}

\section{REFERENCES}

1 Takeda, Y. and Keiichi, N. Emission characteristics of premixed lean diesel combustion with extremely early staged fuel injection. SAE paper 961163, 1996.

2 Harada, A., Shimazaki, N., Sasaki, S., Miyamoto, T., Akagawa, H., and Tsujimura, K. The effects of mixture formation on premixed lean diesel combustion. SAE paper 980533, 1998.

3 Iwabuchi, Y., Kawai, K., Shoji, T., and Takeda, Y. Trial of new concept diesel combustion system premixed compression ignited combustion. SAE paper 1999-01-0185, 1999.

4 Dec, J. E. Advanced compression-ignition engines - understanding the in-cylinder processes. Proc. Combust. Inst., 2009, 32, 2727-2742.

5 Shaver, G. M., Roelle, M. J., Caton, P. A., Kaahaaina, N. B., Ravi, N., Hathout, J.-P., Ahmed, J., Kojic, A., Park, S., Edwards, C. F., and Gerdes, J. C. A physics-based approach to the control of homogeneous charge compression ignition engines with variable valve actuation. Int. J. Eng. Res., 2005, 6(4), 361-375. DOI: 10.1243/146808705 X30512.

6 Bengtsson, J., Strandh, P., Johansson, R., Tunestål, P., and Johansson, B. Hybrid control of homogeneous charge compression ignition (HCCI) engine dynamics. Int. J. Control, 2006, 79, 422-448.

7 Narayanaswamy, K. and Rutland, C. J. A modeling investigation of combustion control variables during DI-diesel HCCI engine transients. SAE paper 2006-01-1084, 2006.

8 Hillion, M., Chauvin, J., Grondin, O., and Petit, N. Active combustion control of diesel HCCI engine: combustion timing. SAE paper 2008-01-0984, 2008.

9 Hillion, M., Buhlbuck, H., Chauvin, J., and Petit, N. Combustion control of diesel engines using injection timing. SAE paper 2008-01-0367, 2009.

10 Nakayama, S., Ibuki, T., Hosaki, H., and Tominaga, $\mathbf{H}$. An application of a model based combustion control to transient cycle-by-cycle diesel combustion. SAE paper 2008-01-1311, 2008.

11 Turin, R., Zhang, R., and Chang, M.-F. Systematic model-based engine control design. SAE paper 2008-01-0994, 2008.

12 Narayanaswamy, K., Hessel, R. P., and Rutland, C. J. A new approach to model DI-diesel HCCI combustion for use in cycle simulation studies. SAE paper 2005-01-3743, 2005.

13 Shaver, G. M., Gerdes, J. C., Roelle, M. J., Caton, P. A., and Edwards, C. F. Dynamic modeling of residual-affected homogeneous charge compres- sion ignition engines with variable valve actuation. J. Dyn. Syst., Meas., Control, 2005, 127, 374-381.

14 Shaver, G. M., Roelle, M. J., and Gerdes, J. C. Modeling cycle-to-cycle dynamics and mode transition in HCCI engines with variable valve actuation. Control Engng Pract., 2006, 14, 213-222.

15 Albrecht, A., Grondin, O., Le Berr, F., and Le Solliec, G. Towards a stronger simulation support for engine control design: a methodological point of view. Oil Gas Sci. Technol. - Rev. IFP, 2007, 62, 437-456.

16 Bengtsson, J., Strandh, P., Johansson, R., Tunestål, P., and Johansson, B. Hybrid modelling of homogeneous charge compression ignition (HCCI) engine dynamics - a survey. Int. J. Control, 2007, 80, 1814-1847.

17 Pope, S. B. PDF methods for turbulent reactive flows. Prog. Energy Combust. Sci., 1985, 11, 119-192.

18 Reitz, R. and Rutland, C. J. Development and testing of diesel engine CFD models. Prog. Energy Combust. Sci., 1995, 21, 173-196.

19 Barths, H., Hasse, C., and Peters, N. Computational fluid dynamics modelling of non-premixed combustion in direct injection diesel engines. Int. J. Eng. Res., 2000, 1(3), 249-267. DOI: 10.1243/ 1468087001545164.

20 Colin, O. and Benkenida, A. The 3-zones extended coherent flame model (ECFM3Z) for computing premixed/diffusion combustion. Oil and Gas Sci. Technol. - Rev. IFP, 2004, 59, 593-609.

21 Aceves, S. M., Flowers, D. L., Martinez-Frias, J., Smith, J. R., Westbrook, C. K., Pitz, W., Dibble, R., Christensen, M., and Johansson, B. A multi-zone model for prediction of HCCI combustion and emissions. SAE paper 2000-01-0327, 2000.

22 Aceves, S. M., Flowers, D. L., Martinez-Frias, J., Smith, J. R., Westbrook, C. K., Pitz, W., Dibble, R., Wright, J. F., Akinyemi, W. C., and Hessel, R. P. A sequential fluid-mechanic chemical kinetic model of propane HCCI combustion. SAE paper 2001-011027, 2001.

23 Babajimopoulos, A., Lavoie, G. A., and Assanis, D. N. Modelling HCCI combustion with high levels of residual gas fraction - a comparison of two VVA strategies. SAE paper 2003-01-3220, 2003.

24 Babajimopoulos, A., Assanis, D. N., Flowers, D. L., Aceves, S. M., and Hessel, R. P. A fully coupled computational fluid dynamics and multi-zone model with detailed chemical kinetics for the simulation of premixed charge compression ignition engines. Int. J. Eng. Res., 2005, 6(5), 497-512. DOI: $10.1243 / 146808705 X 30503$.

25 Flowers, D. L., Aceves, S. M., and Babajimopoulos, A. Effects of charge non-uniformity on heat release and emissions in PCCI engine combustion. SAE paper 2006-01-1363, 2006.

26 Hessel, R. P., Foster, D. E., Aceves, S. M., Davisson, M., Espinosa-Loza, F. J., Flowers, D. L., Pitz, W., Dec, J. E., Sjoberg, M., and Babajimopoulos, A. Modeling iso-octane HCCI using CFD with multizone detailed chemistry; comparison to detailed 
specification data over a range of lean equivalence ratios. SAE paper 2008-01-0047, 2008.

27 Hergart, C.-A., Barths, H., and Siewert, R. M. Modeling approaches for partially premixed compression ignition combustion. SAE paper 2005-010218, 2005.

28 Felsch, C., Sloane, T., Han, J., Barths, H., and Lippert, A. M. Numerical investigation of recompression and fuel reforming in a SIDI-HCCI engine. SAE paper 2007-01-1878, 2007.

29 Petzold, L. R., A description of DASSL: a differential/algebraic system solver, Sandia report SAND82-8637, Sandia National Laboratories, 1982.

30 Peters, N., Paczko, G., Seiser, R., and Seshadri, K. Temperature cross-over and non-thermal runaway at two-stage ignition of n-heptane. Combust. Flame, 2002, 28, 38-59.

31 Heywood, J. B. Internal engine combustion fundamentals, 1988 (McGraw-Hill, New York).

32 Ferziger, J. H. and Peric, M. Computational methods for fluid dynamics, 2002 (Springer, Berlin).

33 Khalighi, B., El Thary, S. H., Haworth, D. C., and Huebler, M. S. Computation and measurement of flow and combustion in a four-valve engine with intake variations. SAE paper 950287, 1995.

34 Ewald, J., Freikamp, F., Paczko, G., Weber, J., Haworth, D. C., and Peters, N., GMTEC: GMTEC developer's manual, Technical report, Advanced Combustion GmbH, 2003.

35 Spiekermann, P., Jerzembeck, S., Felsch, C., Vogel, S., Gauding, M., and Peters, N. Experimental data and numerical simulation of common-rail ethanol sprays at diesel engine-like conditions. Atomiz. Sprays, 2009, 19, 357-386.

36 Peters, N. Turbulent combustion, 2000 (Cambridge Universty Press, Cambridge).

37 Barths, H., Felsch, C., and Peters, N. Mixing models for the two-way-coupling of CFD codes and zero-dimensional multi-zone codes to model HCCI combustion. Combust. Flame, 2009, 156, 130-139.

38 Felsch, C., Dahms, R., Glodde, B., Vogel, S., Jerzembeck, S., Peters, N., Barths, H., Sloane, T., Wermuth, N., and Lippert, A. M. An interactively coupled CFD-multi-zone approach to model HCCI combustion. Flow, Turbul. Combust., 2009, 82, 621-641.

39 Peters, N. Laminar diffusion flamelet models in non-premixed turbulent combustion. Prog. Energy Combust. Sci., 1984, 10, 319-339.

40 Peters, N. Laminar flamelet concepts in turbulent combustion. Symposium (International) on Combustion, 1986, 21, 1231-1250.

41 Pitsch, H., Barths, H., and Peters, N. Threedimensional modeling of $\mathrm{NO}_{x}$ and soot Formation in DI-diesel engines using detailed chemistry based on the interactive flamelet approach. SAE paper 962057, 1996.

42 Hasse, C. and Peters, N. A two mixture fraction flamelet model applied to split injections in a DI diesel engine. Proc. Combust. Inst., 2005, 30, 2755-2762.

43 Felsch, C., Gauding, M., Hasse, C., Vogel, S., and Peters, N. An extended flamelet model for multiple injections in DI diesel engines. Proc. Combust. Inst., 2009, 32, 2775-2783.

44 Woschni, G. A universally applicable equation for instantaneous heat transfer coefficients in the internal combustion engine. SAE paper 670931, 1967.

45 Baehr, H. D. and Stephan, K. Wärme- und Stoffübertragung, 2006 (Springer, Berlin).

46 Vanegas, A., Won, H., Felsch, C., Gauding, M., and Peters, N. Experimental investigation of the effect of multiple injections on pollutant formation in a common-rail DI diesel engine. SAE paper 2008-011191, 2008.

47 Weber, J., Spiekermann, P., and Peters, N. Model calibration for spray penetration and mixture formation in a high-pressure fuel spray using a micro-genetic algorithm and optical data. SAE paper 2005-01-2099, 2005.

48 Felsch, C., Vanegas, A., Kerschgens, B., Peters, N., Hoffmann, K., Drews, P., Abel, D., and Barths, $\mathbf{H}$. Systematic reduction of an interactively coupled CFD-multi-zone approach towards a stand-alone multi-zone model for PCCI combustion. Thiesel 2008: Thermo-and fluid dynamic processes in diesel engines, Universidad Politécnica de Valencia, Valencia, Spain, 2008.

49 Ricardo Software. WAVE basic manual, documentation/user's manual, version 5.0, Technical report, Ricardo Software, 2003.

50 Gamma Technologies. GT-POWER user's manual and tutorial, GT-SUITE version 7.0, Technical report, Gamma Technologies, 2008.

51 Richert, F. Objektorientierte Modellbildung und Nichtlineare Prädiktive Regelung von Dieselmotoren. PhD Thesis, RWTH Aachen University, Germany, 2005.

52 Hoffmann, K., Drews, P., Abel, D., Felsch, C., Vanegas, A., and Peters, N. A cycle-based multizone simulation approach including cycle-to-cycle dynamics for the development of a controller for PCCI combustion. SAE paper 2009-01-0671, 2009.

53 Nelles, O. Nonlinear system identification: from classical approaches to neural networks and fuzzy models, 2000 (Springer, Berlin).

54 Peréz, E., Blasco, X., García-Nieto, S., and Sanchis, J. Diesel engine identification and predictive control using Wiener and Hammerstein models. In Proceedings of the 2006 IEEE International Conference on Control Applications, Munich, Germany, 2006 (IEEE, Piscataway, NJ).

55 Schulze, T., Wiedemeier, M., and Schuette, $\mathbf{H}$. Crank angle - based diesel engine modeling for hardware-in-the-loop applications with in-cylinder pressure sensors. SAE paper 2007-01-1303, 2007.

56 ETAS GmbH. ES 1000.3 Benutzerhandbuch, technical report, ETAS GmbH, 2008. 
57 Guzzella, L. and Onder, C. H. Introduction to modeling and control of internal combustion engine systems, 2004 (Springer, Berlin).

58 IAV GmbH Ingenieursgesellschaft Auto und Verkehr. FI2RE - flexible injection and ignition for rapid engineering, 2008, available from http://www.iav. com/de/1_engineering/powertrainmechatronik/ messtechnik/fi2re.php.

59 Lunze, J. Regelungstechnik 2: Mehrgrößensysteme, Digitale Regelung, 2008 (Springer, Berlin).

60 The MathWorks, Inc. Matlab 7.6 manual (R2008a). Technical report, The MathWorks, Inc., 2008.
61 Eng, J. A. Characterization of pressure waves in HCCI combustion. SAE paper 2002-01-2859, 2002.

62 RWTH Aachen University and Bielefeld University. SFB 686 - Modellbasierte Regelung der homogenisierten Niedertemperatur-Verbrennung, available from http://www.sfb686.rwth-aachen.de, 2006.

63 Drews, P., Hoffmann, K., Beck, R., Gasper, R., Vanegas, A., Felsch, C., Peters, N., and Abel, D. Fast model predictive control for the air path of a turbocharged diesel engine. European Control Conference 2009, Budapest, Hungary, 2009. 